International Electronic Journal of Algebra

VOLUME 32 (2022) 1-45

DOI: $10.24330 /$ ieja.1060709

\title{
WEAK STUFFLE ALGEBRAS
}

\author{
Cécile Mammez \\ Received: 26 September 2020; Revised: 24 March 2021; Accepted: 15 April 2021 \\ Communicated by Abdullah Harmancı
}

\begin{abstract}
Motivated by $q$-shuffle products determined by Singer from $q$ analogues of multiple zeta values, we build in this article a generalisation of the shuffle and stuffle products in terms of weak shuffle and stuffle products. Then, we characterise weak shuffle products and give as examples the case of an alphabet of cardinality two or three. We focus on a comparison between algebraic structures respected in the classical case and in the weak case. As in the classical case, each weak shuffle product can be equipped with a dendriform structure. However, they have another behaviour towards the quadri-algebra and the Hopf algebra structure. We give some relations satisfied by weak stuffle products.
\end{abstract}

Mathematics Subject Classification (2020): 05A05, 05E40, 16T30, 68R15

Keywords: Shuffle algebra, stuffle algebra, dendriform algebra, quadri-algebra, Hopf algebra

\section{Introduction}

The notion of shuffle and stuffle algebras is widely used in several fields of mathematics. Indeed, they participate in the study of Rota-Baxter algebras with the notion of mixable shuffle algebras $[6,14,20]$, in the study of Yang-Baxter algebras [21], in the study of quasi-symmetric functions and words algebras $[4,5,12,13,24,25$, $26,33]$, in the study of multiple zeta values $[7,8,15,16,17,18,19,30,34] \ldots$

The classical stuffle product comes from the product of classical multiple zeta values and is defined by the relation

$$
a u \square b v=a(u \square b v)+b(a u \square v)+(a \diamond b)(u \square v)
$$

where $a$ and $b$ are letters, $u$ and $v$ are words and $\diamond$ is an associative and commutative product which is equal to 0 in the case of the classical shuffle product. Thus, the shuffle part of the relation is symmetric and does not depend on letters of any words in the product. In his work, Singer focuses on $q$-shuffle products coming from $q$-analogues of multiples zeta values. This case enables the existance of some 
letters $p$ and $y$ satisfying a relation in the form of

$$
y u \square p v=p v \square y u=y(u \square p v)
$$

for any words $u$ and $v$. This new $q$-shuffle relation is not symmetric and depends on the beginning of each word in the product. This leads to focus on new generalisations of shuffle and stuffle products $[7,8,31]$.

In this article, we present a new generalisation of shuffle and stuffle algebras, we study their algebraic structures and compare them to the classical case. The article is organised as follows.

- In Section 2, we recall the classical notion of shuffle and stuffle product thanks to the multiple zeta values as well as the calculation by Singer of $q$-shuffle associated to the Schlesinger-Zudilin model and the Bradley-Zhao model.

- In Section 3, we define a generalisation of the classical shuffle product and the classical stuffle product called weak shuffle products and weak stuffle products and prove a characterisation of weak shuffle products. We detail the case of an alphabet of cardinality 2 or 3 .

- In Section 4, we focus on algebraic structures respected by the classical shuffle product and we determine if the weak shuffle products respect them too. Thus we prove that weak shuffle products are dendriform but there are obstacles to the quadri-algebra structure.

- In Section 5, we express some relations satistied by weak stuffle products and we express the $q$-shuffle products given by Singer in terms of weak stuffle product. Besides, in the case of an infinite, countable and totally ordered alphabet $\left\{x_{1}, \ldots, x_{n}, \ldots\right\}$, we prove that, if the contracting part in the weak stuffle products is expressed as $f_{3}\left(x_{i} \otimes x_{j}\right) \in \mathbb{K}^{*} x_{i+j}$, then the shuffle part is the null product or the classical shuffle product. We give some informations more about weak stuffle products in the case of an alphabet of cardinality 2 or 3 .

- In Section 6, we prove that a weak stuffle product is compatible with the deconcatenation coproduct if and only if the underlying weak shuffle product is the classical shuffle product and the contracting part is associative and commutative.

- Computation programs used to prove Lemma 3.17 are detailed in Section 7. 


\section{Reminders}

2.1. Classical shuffle and stuffle algebras. We recall here the definition of the stuffle product in the context of the multiple zeta values.

Definition 2.1. Let $s$ be an integer and let $\left(k_{1}, \ldots, k_{s}\right)$ be an $s$-tuple in $\mathbb{N}_{\geq 2} \times \mathbb{N}^{s-1}$. The multiple zeta value associated to $\left(k_{1}, \ldots, k_{s}\right)$ is

$$
\zeta\left(k_{1}, \ldots, k_{s}\right)=\sum_{\substack{\left(m_{1}, \ldots, m_{s}\right) \in \mathbb{N} \\ m_{1}>\ldots>m_{s}>0}} \frac{1}{m_{1}^{k_{1}} \ldots m_{s}^{k_{s}}} .
$$

On multiple zeta values, we consider the product of functions taking values in C. For instance,

$$
\begin{aligned}
\zeta(n) \zeta(m) & =\zeta(m, n)+\zeta(n, m)+\zeta(m+n) \\
\zeta(n, p) \zeta(m) & =\zeta(m, n, p)+\zeta(n, m, p)+\zeta(n, p, m)+\zeta(n+m, p)+\zeta(n, p+m) .
\end{aligned}
$$

Then, it leads to the following algebraic definition and following theorem [15].

Theorem 2.2. Let $X=\left\{x_{1}, \ldots, x_{n}, \ldots\right\}$ be a countable alphabet. Let $\mathbb{K}\langle X\rangle$ be the algebra of words on the alphabet $X$. We define the product $\star$, called the stuffle product, by:

$$
\begin{aligned}
u \star 1 & =1 \star u=1, \\
u \star 0 & =0 \star u=0, \\
x_{i} u \star x_{j} v & =x_{i}\left(u \star x_{j} v\right)+x_{j}\left(x_{i} u \star v\right)+x_{i+j}(u \star v)
\end{aligned}
$$

for any letters $x_{i}$ and $x_{j}$ and any words $u$ and $v$.

Then

$$
\begin{aligned}
x_{i} u x_{k} \star x_{j} v x_{l} & =x_{i}\left(u x_{k} \star x_{j} v x_{l}\right)+x_{j}\left(x_{i} u x_{k} \star v x_{l}\right)+x_{i+j}\left(u x_{k} \star v x_{l}\right) \\
& =\left(x_{i} u \star x_{j} v x_{l}\right) x_{k}+\left(x_{i} u x_{k} \star x_{j} v\right) x_{l}+\left(x_{i} u \star x_{j} v\right) x_{k+l}
\end{aligned}
$$

and $(\mathbb{K}\langle X\rangle, \star)$ is an associative and commutative algebra.

It is possible to define another algebra:

Theorem 2.3. Let $X=\left\{x_{1}, \ldots, x_{n}, \ldots\right\}$ be a countable alphabet. Let $\mathbb{K}\langle X\rangle$ be the algebra of words on the alphabet $X$. We define the product $\omega$, called the shuffle product, by:

$$
\begin{aligned}
& u \amalg 1=1 ш u=1, \\
& u \amalg 0=0 \amalg u=0, \\
& x_{i} u \amalg x_{j} v=x_{i}\left(u \amalg x_{j} v\right)+x_{j}\left(x_{i} u \amalg v\right)
\end{aligned}
$$


for any letters $x_{i}$ and $x_{j}$ and any words $u$ and $v$.

Then

$$
\begin{aligned}
x_{i} u x_{k} \uplus x_{j} v x_{l} & =x_{i}\left(u x_{k} \amalg x_{j} v x_{l}\right)+x_{j}\left(x_{i} u x_{k} \amalg v x_{l}\right) \\
& =\left(x_{i} u \amalg x_{j} v x_{l}\right) x_{k}+\left(x_{i} u x_{k} \amalg x_{j} v\right) x_{l}
\end{aligned}
$$

and $(\mathbb{K}\langle X\rangle, \Psi)$ is an associative and commutative algebra.

Theorem 2.4. Let $X=\left\{x_{1}, \ldots, x_{n}, \ldots\right\}$ be a countable alphabet. The algebras $(\mathbb{K}\langle X\rangle, \star)$ and $(\mathbb{K}\langle X\rangle, \omega)$ are isomorphic.

Proof. This theorem was proved by Hoffman [16, Theorem 2.5] by describing an explicit isomorphism exp. Another construction of exp leading to the proof of this theorem is given in [26, Proposition 41].

2.2. $q$-shuffle products for the Schlesinger-Zudilin model and the Bradley-

Zhao model. Let $q$ be a real number such that $0<q<1$. A $q$-analogue of a positive integer $m$ is defined by

$$
[m]_{q}=\frac{1-q^{m}}{1-q}=1+q+\cdots+q^{m-1} .
$$

The Schlesinger-Zudilin model $[28,36]$ is defined as the following $q$-sum:

$$
\begin{aligned}
\zeta_{q}^{S Z}\left(k_{1}, \ldots, k_{n}\right) & =(1-q)^{-\left(k_{1}+\cdots+k_{n}\right)} \sum_{\begin{array}{c}
\left(m_{1}, \ldots, m_{s}\right) \in \mathbb{N} \\
m_{1}>\cdots>m_{s}>0
\end{array}} \frac{q^{m_{1} k_{1}+\cdots+m_{n} k_{n}}}{\left[m_{1}\right]_{q}^{k_{1}} \ldots\left[m_{n}\right]_{q}^{k_{n}}} \\
& =\sum_{\substack{\left(m_{1}, \ldots, m_{s}\right) \in \mathbb{N} \\
m_{1}>\cdots>m_{s}>0}} \frac{q^{m_{1} k_{1}+\cdots+m_{n} k_{n}}}{\left(1-q^{m_{1}}\right)^{k_{1}} \ldots\left(1-q^{m_{n}}\right)^{k_{n}}}
\end{aligned}
$$

for any $\left(k_{1}, \ldots, k_{n}\right) \in\left(\mathbb{N}^{*}\right)^{n}$.

The Bradley-Zhao model $[2,35]$ is defined as the following $q$-sum:

$$
\begin{aligned}
\zeta_{q}^{B Z}\left(k_{1}, \ldots, k_{n}\right)= & (1-q)^{-\left(k_{1}+\cdots+k_{n}\right)} \sum_{\substack{\left(m_{1}, \ldots, m_{s}\right) \in \mathbb{N} \\
m_{1}>\cdots>m_{s}>0}} \frac{q^{m_{1}\left(k_{1}-1\right)+\cdots+m_{n}\left(k_{n}-1\right)}}{\left[m_{1}\right]_{q}^{k_{1}} \ldots\left[m_{n}\right]_{q}^{k_{n}}} \\
= & \sum_{\substack{\left(m_{1}, \ldots, m_{s}\right) \in \mathbb{N} \\
m_{1}>\cdots>m_{s}>0}} \frac{q^{m_{1}\left(k_{1}-1\right)+\cdots+m_{n}\left(k_{n}-1\right)}}{\left(1-q^{m_{1}}\right)^{k_{1}} \ldots\left(1-q^{m_{n}}\right)^{k_{n}}}
\end{aligned}
$$

for any $\left(k_{1}, \ldots, k_{n}\right) \in \mathbb{N}^{n}$ with $k_{1} \geq 2$.

From those two models, Singer defined two $q$-shuffle products corresponding to the algebraic version of the Schlesinger-Zudilin model and the Bradley-Zhao model and proved the following two theorems in $[29,30,31]$ : 
Theorem 2.5 (Singer). Let $X=\{y, p\}$ be an alphabet. The q-shuffle product associated to the Schlesinger-Zudilin model is given by: for any words $u$ and $v$,

(1) $1 \varpi_{S Z} u=u \varpi_{S Z} 1=u$,

(2) $y u \varpi_{S Z} v=v \varpi_{S Z} y u=y\left(u \varpi_{S Z} v\right)$,

(3) $p u \varpi_{S Z} p v=p\left(u \varpi_{S Z} p v\right)+p\left(p u \varpi_{S Z} v\right)+p\left(u \varpi_{S Z} v\right)$.

Besides, it is an associative and commutative product.

Theorem 2.6 (Singer). Let $X=\{y, p, \bar{p}\}$ be an alphabet. The $q$-shuffle product associated to the Bradley-Zhao model is given by: for any words $u$ and $v$,

(1) $1 \varpi_{B Z} u=u \varpi_{B Z} 1=u$,

(2) $y u \varpi_{B Z} v=v \varpi_{B Z} y u=y\left(u \varpi_{B Z} v\right)$,

(3) $a u \varpi_{B Z} b v=a\left(u \varpi_{B Z} b v\right)+b\left(a u \varpi_{B Z} v\right)+[a, b] a\left(u \varpi_{B Z} v\right)$ where

$$
a, b \in\{p, \bar{p}\},[p, p]=-[\bar{p}, \bar{p}]=1 \text { and }[p, \bar{p}]=[\bar{p}, p]=0 .
$$

Besides, it is an associative and commutative product.

\section{Definition and characterisation of weak shuffle products}

The aim of this section is to define a generalisation of the classical shuffle product, the classical stuffle product, and the two $q$-shuffle products given by the SchlesingerZudilin model and the Bradley-Zhao model. We give and prove a characterisation of weak shuffle products too. Then we explicit the case of an alphabet of cardinality 2 or 3.

\subsection{Characterisation.}

Definition 3.1. An alphabet is a non-empty finite or countable set $X$.

Definition 3.2. Let $X$ be an alphabet. We denote by $X^{*}$ the set of words on the alphabet $X$ and by $\mathbb{K}\langle X\rangle$ the tensor algebra generated by $X$ (i.e. the algebra of words on $X)$. The space $\mathbb{K}\langle X\rangle$ is graded by the length of words.

Definition 3.3. Let $X$ be an alphabet. A weak stuffle product on $\mathbb{K}\langle X\rangle$ is an associative and commutative product $\square$ such that for any $(a, b) \in(X)^{2}$ and any $(u, v) \in\left(X^{*}\right)^{2}$

$$
\begin{aligned}
u \square 1 & =1 \square u=u, \\
u \square 0 & =0 \square u=0, \\
a u \square b v & =f_{1}(a \otimes b) a(u \square b v)+f_{2}(a \otimes b) b(a u \square v)+f_{3}(a \otimes b)(u \square v)
\end{aligned}
$$

where

(1) $f_{1}$ and $f_{2}$ are linear maps from $\mathbb{K} . X \otimes \mathbb{K} . X$ to $\mathbb{K}$, 
(2) $f_{3}=k g$ is a linear map from $\mathbb{K} . X \otimes \mathbb{K} . X$ to $\mathbb{K} . X$ such that $k(a \otimes b) \in \mathbb{K}$ and $g(a \otimes b) \in X$ for any $(a, b) \in X^{2}$,

(3) If $f_{3} \equiv 0$ then the product $\square$ is called a weak shuffle product.

Examples 3.4. Let $X=\left\{x_{1}, \ldots, x_{n}, \ldots\right\}$ be an infinite alphabet.

(1) The classical shuffle product on $\mathbb{K}\langle X\rangle$ is a weak stuffle product where $f_{1}(a \otimes$ $b)=1$ and $f_{2}(a \otimes b)=1$ for any $(a, b) \in X^{2}$, and $f_{3} \equiv 0$.

(2) The classical stuffle product on $\mathbb{K}\langle X\rangle$ is a weak stuffle product where $f_{1}(a \otimes$ $b)=1$ and $f_{2}(a \otimes b)=1$ for any $(a, b) \in X^{2}$, and $f_{3}\left(x_{i} \otimes x_{j}\right)=x_{i+j}$ for any $(i, j) \in\left(\mathbb{N}^{*}\right)^{2}$.

(3) The stuffle product on $\mathbb{K}\langle X\rangle$ given by Hoffman and Ihara in [18] is a weak stuffle product where $f_{1}(a \otimes b)=1$ and $f_{2}(a \otimes b)=1$ for any $(a, b) \in X^{2}$, and $f_{3}\left(x_{i} \otimes x_{j}\right)=-x_{i+j}$ for any $(i, j) \in\left(\mathbb{N}^{*}\right)^{2}$.

Theorem 3.5. Let $\square$ be a product on $\mathbb{K}\langle X\rangle$. The map $\square$ is a weak shuffle product if and only if, for any distinct letters $a, b$, and $c$ in $X$ :

(1) $f_{1}(a \otimes b)=f_{2}(b \otimes a)$.

(2) (a) either $f_{1}(a \otimes a)=f_{2}(a \otimes a)=\alpha$ with $\alpha \in\{0,1\}$ and

(i) $f_{1}(a \otimes b) f_{1}(b \otimes a)\left[f_{1}(a \otimes a)-1\right]=0$,

(ii) $f_{1}(a \otimes a) f_{1}(a \otimes b)\left[f_{1}(a \otimes b)-1\right]=0$,

(iii) $f_{1}(a \otimes a) f_{1}(b \otimes a)\left[f_{1}(b \otimes a)-1\right]=0$.

(b) or $f_{1}(a \otimes a)=\alpha, f_{2}(a \otimes a)=1-\alpha$ with $\alpha \in \mathbb{R}$ and

(i) $f_{1}(a \otimes b)=1$,

(ii) $f_{1}(b \otimes a)=0$.

(3) $f_{1}(a \otimes b) f_{1}(b \otimes c)\left[f_{1}(a \otimes c)-1\right]=0$.

(4) $f_{3} \equiv 0$.

Remark 3.6. It is sometimes usefull to use in calculations the following statement induced by the item $(2)(b)$ of the Theorem 3.5:

"If $f_{1}(a \otimes b)=0$ or $f_{1}(b \otimes a) \neq 0$ then $f_{1}(a \otimes a)=f_{2}(a \otimes a)=\alpha$ with $\alpha \in\{0,1\}$ ".

Proof. Let us prove first the direct implication. Let us assume $\square$ is a weak shuffle product. Let $a, b$, and $c$ be three distinct letters. Then, by direct calculations,

(A) $a \square b=b \square a$ gives relation $f_{1}(a \otimes b)=f_{2}(b \otimes a)$.

(B) $a \square a a=a a \square a$ gives $f_{1}(a \otimes a)=f_{2}(a \otimes a)$ or $f_{1}(a \otimes a)=1-f_{2}(a \otimes a)$.

(C) $a \square a b=a b \square a$ gives, if $f_{1}(a \otimes b)=0$ or $f_{1}(b \otimes a) \neq 0$, that $f_{1}(a \otimes a)=$ $f_{2}(a \otimes a)$. Thus, if $f_{1}(a \otimes a)=1-f_{2}(a \otimes a)$ and $f_{1}(a \otimes a) \neq \frac{1}{2}$ then $f_{1}(a \otimes b) \neq 0$ and $f_{1}(b \otimes a)=0$. The relation $a \square a b=a b \square a$ implies $f_{1}(a \otimes b)=1$. 
(D) $(a \square a) \square b=a \square(a \square b)=(a \square b) \square a$ with $f_{1}(a \otimes a)=f_{2}(a \otimes a)$ give

(a) $f_{1}(a \otimes b) f_{1}(b \otimes a)\left[f_{1}(a \otimes a)-1\right]=0$,

(b) $f_{1}(a \otimes a) f_{1}(a \otimes b)\left[f_{1}(a \otimes b)-1\right]=0$,

(c) $f_{1}(a \otimes a) f_{1}(b \otimes a)\left[f_{1}(b \otimes a)-1\right]=0$.

(E) $(a \square b) \square c=a \square(b \square c)$ gives $f_{1}(a \otimes b) f_{1}(b \otimes c)\left[f_{1}(a \otimes c)-1\right]=0$.

(F) $(a \square a) \square a b=a \square(a \square a b)$ implies that if $f_{1}(a \otimes a)=1-f_{2}(a \otimes a)=\frac{1}{2}$ then $f_{1}(a \otimes b)=1$ and $f_{1}(b \otimes a)=0$.

(G) $(a \square a) \square a a=a \square(a \square a a)$ and $(a \square a) \square a a a=a \square(a \square a a a)$ implies that if $f_{1}(a \otimes$ $a)=f_{2}(a \otimes a)=\alpha$ then $\alpha \in\left\{0,1, \frac{1}{2}\right\}$.

(H) Cases $b a \square a=a \square b a, a a \square b=b \square a a, a b \square c=c \square a b$ and $(a \square a) \square a=a \square(a \square a)$ do not give any further relations.

As a consequence, in the Theorem 3.5,

- the item (1) is proved by the item (A),

- the item (2)(a) is proved by the items (B), (D), (F) and (G),

- the item (2) (b) is proved by the items (B), (C) and (F),

- the item (3) is proved by the item (E),

- the item (4) is satisfied by the definition of a weak shuffle product.

Conversly, if $\square$ satisfies all relations given in Theorem 3.5 then for any couple $(u, v)$ and any triple $\left(w_{1}, w_{2}, w_{3}\right)$ of words such that length $(u)+\operatorname{length}(v) \leq 3$ and length $\left(w_{1}\right)+$ length $\left(w_{2}\right)+$ length $\left(w_{3}\right) \leq 3$ one has: $u \square v=v \square u$ and $\left(w_{1} \square w_{2}\right) \square w_{3}=$ $w_{1} \square\left(w_{2} \square w_{3}\right)$.

We assume now there exists an integer $n \geq 3$ such that $u \square v=v \square u$ and $\left(w_{1} \square w_{2}\right) \square w_{3}=w_{1} \square\left(w_{2} \square w_{3}\right)$ for any words $u, v, w_{1}, w_{2}$ with length $(u)+\operatorname{length}(v) \leq$ $n$ and length $\left(w_{1}\right)+$ length $\left(w_{2}\right)+$ length $\left(w_{3}\right) \leq n$.

Let now $u$ and $v$ be two words $\operatorname{such}$ that length $(u)+\operatorname{length}(v)=n+1$. Then there exist two letters $a$ and $b$ and two words $w_{1}$ and $w_{2}$ (not necessarily non-empty) such that $u=a w_{1}$ and $v=b w_{2}$. Then, by induction, we get:

case $a \neq b$ :

$$
\begin{aligned}
u \square v & =f_{1}(a \otimes b) a\left(w_{1} \square b w_{2}\right)+f_{1}(b \otimes a) b\left(a w_{1} \square w_{2}\right) \\
& =f_{1}(a \otimes b) a\left(b w_{2} \square w_{1}\right)+f_{1}(b \otimes a) b\left(w_{2} \square a w_{1}\right)=v \square u .
\end{aligned}
$$

case $a=b$ and $f_{1}(a \otimes a)=f_{2}(a \otimes a)$ :

$$
\begin{aligned}
u \square v & =f_{1}(a \otimes a) a\left(w_{1} \square a w_{2}\right)+f_{1}(a \otimes a) a\left(a w_{1} \square w_{2}\right) \\
& =f_{1}(a \otimes a) a\left(a w_{2} \square w_{1}\right)+f_{1}(a \otimes a) a\left(w_{2} \square a w_{1}\right)=v \square u .
\end{aligned}
$$

case $a=b$ and $f_{2}(a \otimes a)=1-f_{1}(a \otimes a)$ : There exist two words $w_{3}$ and $w_{4}$, not necessarily non-empty, not starting by $a$ and two positive integers $k$ and $l$ 
such that $w_{1}=\underbrace{a \ldots a}_{k \text { times }} w_{3}$ and $w_{2}=\underbrace{a \ldots a}_{l \text { times }} w_{4}$. First of all, by induction,

$$
\underbrace{a \ldots a}_{k \text { times }} \square \underbrace{a \ldots a}_{l \text { times }}=\underbrace{a \ldots a}_{k+l \text { times }} .
$$

Besides, relations satisfied by $\square$ enjoin $f_{1}(a \otimes c)=1$ and $f_{2}(c \otimes a)=0$ for any letter $c \neq a$. So,

$$
u \square v=(\underbrace{a \ldots a}_{k \text { times }} \square \underbrace{a \ldots a}_{l \text { times }})\left(w_{3} \square w_{4}\right)=(\underbrace{a \ldots a}_{l \text { times }} \square \underbrace{a \ldots a}_{k \text { times }})\left(w_{4} \square w_{3}\right)=v \square u .
$$

As a consequence, $\square$ is a commutative product.

Let now $w_{1}, w_{2}$ and $w_{3}$ be three words such that length $\left(w_{1}\right)+\operatorname{length}\left(w_{2}\right)+$ length $\left(w_{3}\right)=n+1$. Then there exist three letters $a, b$ and $c$ and three words $w_{4}, w_{5}$ and $w_{6}$ (not necessarily non-empty) such that $w_{1}=a w_{4}, w_{2}=b w_{5}$ and $w_{3}=c w_{6}$. Then, by induction, we get:

case $a, b$ and $c$ distinct:

$$
\begin{aligned}
\left(w_{1} \square w_{2}\right) \square w_{3} & =f_{1}(a \otimes b) f_{1}(a \otimes c) a\left[\left(w_{4} \square b w_{5}\right) \square c w_{6}\right]+f_{1}(a \otimes b) f_{1}(c \otimes a) c\left[a\left(w_{4} \square b w_{5}\right) \square w_{6}\right] \\
& +f_{1}(b \otimes a) f_{1}(b \otimes c) b\left[\left(a w_{4} \square w_{5}\right) \square c w_{6}\right]+f_{1}(b \otimes a) f_{1}(c \otimes b) c\left[b\left(a w_{4} \square w_{5}\right) \square w_{6}\right]
\end{aligned}
$$

and

$$
\begin{aligned}
w_{1} \square\left(w_{2} \square w_{3}\right) & =f_{1}(b \otimes c) f_{1}(a \otimes b) a\left[w_{4} \square b\left(w_{5} \square c w_{6}\right)\right]+f_{1}(b \otimes c) f_{1}(b \otimes a) b\left[a w_{4} \square\left(w_{5} \square c w_{6}\right)\right] \\
& +f_{1}(c \otimes b) f_{1}(a \otimes c) a\left[w_{4} \square c\left(b w_{5} \square w_{6}\right)\right]+f_{1}(c \otimes b) f_{1}(c \otimes a) c\left[a w_{4} \square\left(b w_{5} \square w_{6}\right)\right] .
\end{aligned}
$$

However

$$
\left(w_{4} \square b w_{5}\right) \square c w_{6}=w_{4} \square\left(b w_{5} \square c w_{6}\right)=f_{1}(b \otimes c) w_{4} \square b\left(w_{5} \square c w_{6}\right)+f_{1}(c \otimes b) w_{4} \square c\left(b w_{5} \square w_{6}\right),
$$$$
a w_{4} \square\left(b w_{5} \square w_{6}\right)=\left(a w_{4} \square b w_{5}\right) \square w_{6}=f_{1}(a \otimes b) a\left(w_{4} \square b w_{5}\right) \square w_{6}+f_{1}(b \otimes a) b\left(a w_{4} \square w_{5}\right) \square w_{6},
$$
and $f_{1}$ satisfies $f_{1}(x \otimes y) f_{1}(y \otimes z)\left(f_{1}(x \otimes z)-1\right)=0$ for any set $\{x, y, z\} \subset$ $X$. Thus,

$$
\left(w_{1} \square w_{2}\right) \square w_{3}=w_{1} \square\left(w_{2} \square w_{3}\right) .
$$

case $a=b$ and $(a \neq c)$ : By commutativity it is the same case as $(a=c$ and $b \neq a)$ or $(b=c$ and $a \neq b)$.

$$
\begin{aligned}
\left(w_{1} \square w_{2}\right) \square w_{3} & =f_{1}(a \otimes a) f_{1}(a \otimes c) a\left[\left(w_{4} \square a w_{5}\right) \square c w_{6}\right]+f_{1}(a \otimes a) f_{1}(c \otimes a) c\left[a\left(w_{4} \square a w_{5}\right) \square w_{6}\right] \\
& +f_{2}(a \otimes a) f_{1}(a \otimes c) a\left[\left(a w_{4} \square w_{5}\right) \square c w_{6}\right]+f_{2}(a \otimes a) f_{1}(c \otimes a) c\left[a\left(a w_{4} \square w_{5}\right) \square w_{6}\right]
\end{aligned}
$$

and

$$
\begin{aligned}
w_{1} \square\left(w_{2} \square w_{3}\right) & =f_{1}(a \otimes c) f_{1}(a \otimes a) a\left[w_{4} \square a\left(w_{5} \square c w_{6}\right)\right]+f_{1}(a \otimes c) f_{2}(a \otimes a) a\left[a w_{4} \square\left(w_{5} \square c w_{6}\right)\right] \\
& +f_{1}(c \otimes a) f_{1}(a \otimes c) a\left[w_{4} \square c\left(a w_{5} \square w_{6}\right)\right]+f_{1}(c \otimes a)^{2} c\left[a w_{4} \square\left(a w_{5} \square w_{6}\right)\right] .
\end{aligned}
$$


However

$\left(w_{4} \square a w_{5}\right) \square c w_{6}=w_{4} \square\left(a w_{5} \square c w_{6}\right)=f_{1}(a \otimes c) w_{4} \square a\left(w_{5} \square c w_{6}\right)+f_{1}(c \otimes a) w_{4} \square c\left(a w_{5} \square w_{6}\right)$,

$a w_{4} \square\left(a w_{5} \square w_{6}\right)=\left(a w_{4} \square a w_{5}\right) \square w_{6}=f_{1}(a \otimes a) a\left(w_{4} \square a w_{5}\right) \square w_{6}+f_{2}(a \otimes a) a\left(a w_{4} \square w_{5}\right) \square w_{6}$,

and $f_{1}$ satisfies

(1) If $f_{1}(a \otimes a)=f_{2}(a \otimes a) \in\{0,1\}$ then

(a) $f_{1}(a \otimes b) f_{1}(b \otimes a)\left[f_{1}(a \otimes a)-1\right]=0$,

(b) $f_{1}(a \otimes a) f_{1}(a \otimes b)\left[f_{1}(a \otimes b)-1\right]=0$,

(c) $f_{1}(a \otimes a) f_{1}(b \otimes a)\left[f_{1}(b \otimes a)-1\right]=0$.

(2) If $f_{1}(a \otimes a)=1-f_{2}(a \otimes a)$ then $f_{1}(a \otimes c)=1$ and $f_{1}(c \otimes a)=0$.

Thus, $\left(w_{1} \square w_{2}\right) \square w_{3}=w_{1} \square\left(w_{2} \square w_{3}\right)$.

case $a=b=c$ and $f_{1}(a \otimes a)=f_{2}(a \otimes a)$ :

$$
\begin{aligned}
\left(w_{1} \square w_{2}\right) \square w_{3} & =f_{1}(a \otimes a)^{2} a\left[\left(w_{4} \square a w_{5}\right) \square a w_{6}\right]+f_{1}(a \otimes a)^{2} a\left[a\left(w_{4} \square a w_{5}\right) \square w_{6}\right] \\
& +f_{1}(a \otimes a)^{2} a\left[\left(a w_{4} \square w_{5}\right) \square a w_{6}\right]+f_{1}(a \otimes a)^{2} a\left[a\left(a w_{4} \square w_{5}\right) \square w_{6}\right]
\end{aligned}
$$

and

$$
\begin{aligned}
w_{1} \square\left(w_{2} \square w_{3}\right) & =f_{1}(a \otimes a)^{2} a\left[w_{4} \square a\left(w_{5} \square a w_{6}\right)\right]+f_{1}(a \otimes a)^{2} a\left[a w_{4} \square\left(w_{5} \square a w_{6}\right)\right] \\
& +f_{1}(a \otimes a)^{2} a\left[w_{4} \square a\left(a w_{5} \square w_{6}\right)\right]+f_{1}(a \otimes a)^{2} a\left[a w_{4} \square\left(a w_{5} \square w_{6}\right)\right] .
\end{aligned}
$$

Thus, $\left(w_{1} \square w_{2}\right) \square w_{3}=w_{1} \square\left(w_{2} \square w_{3}\right)$.

case $a=b=c$ and $f_{2}(a \otimes a)=1-f_{1}(a \otimes a)$ : There exist three words $w_{7}, w_{8}$ and $w_{9}$ not necessarily non-empty, not starting by $a$ and three positive integers $k, l$ and $m$ such that $w_{1}=\underbrace{a \ldots a}_{k \text { times }} w_{7}, w_{2}=\underbrace{a \ldots a}_{l \text { times }} w_{8}$ and $w_{3}=\underbrace{a \ldots a}_{m \text { times }} w_{9}$. Besides, relations satisfied by $\square$ enjoin $f_{1}(a \otimes c)=1$ and $f_{2}(c \otimes a)=0$ for any letter $c \neq a$. So,

$$
\begin{aligned}
\left(w_{1} \square w_{2}\right) \square w_{3} & =[(\underbrace{a \ldots a}_{k \text { times }} \square \underbrace{a \ldots a}_{l \text { times }}) \square \underbrace{a \ldots a}_{k \text { times }}]\left[\left(w_{7} \square w_{8}\right) \square w_{9}\right] \\
& =\underbrace{a \ldots a}_{k+l+m \text { times }}\left[\left(w_{7} \square w_{8}\right) \square w_{9}\right] \\
& =[\underbrace{a \ldots a}_{k \text { times }} \square(\underbrace{a \ldots a}_{l \text { times }} \square \underbrace{a \ldots a}_{k \text { times }})]\left[w_{7} \square\left(w_{8} \square w_{9}\right)\right]=w_{1} \square\left(w_{2} \square w_{3}\right) .
\end{aligned}
$$

Corollary 3.7. Let $\mathbb{K}$ be a field of characteristic 0, let $X$ be a countable alphabet and let $\square$ be a weak shuffle product on $\mathbb{K}\langle X\rangle$.

(1) There exists at most one letter a such that $f_{1}(a \otimes a)=1-f_{2}(a \otimes a)$. 
(2) If there exists a letter a such that $f_{1}(a \otimes a)=1-f_{2}(a \otimes a)$ then, for any word $u$ and $v$, the calculation of $u \square v$ does not depend on the value of $f_{1}(a \otimes a)$.

(3) If $f_{1}(a \otimes b)=f_{1}(b \otimes a)=1$ then $f_{1}(a \otimes a)=f_{2}(a \otimes a)=f_{1}(b \otimes b)=f_{2}(b \otimes b)=$ $1, f_{1}(a \otimes c)=f_{1}(b \otimes c) \in\{0,1\}$ and $f_{1}(c \otimes a)=f_{1}(c \otimes b) \in\{0,1\}$ for any $c \in X \backslash\{a, b\}$.

Proof. (1) If there are two letters $a$ and $b$ such that $a \neq b, f_{1}(a \otimes a)=$ $1-f_{2}(a \otimes a)$ and $f_{1}(b \otimes b)=1-f_{2}(b \otimes b)$ then $1=f_{1}(a \otimes b)=0$ and $0=f_{1}(b \otimes a)=1$. Contradiction.

(2) Let $a$ such that $f_{1}(a \otimes a)=1-f_{2}(a \otimes a)$. If $u$ and $v$ are words in $X^{*} \backslash a X^{*}$, since $f_{1}(a \otimes b)=1$ and $f_{1}(b \otimes a)=0$ for any $b \neq a$, there does not exist any triple $\left(w, u^{\prime}, v^{\prime}\right)$ such that $u \square v=w\left(a u^{\prime} \square a v^{\prime}\right)$.

(3) If $f_{1}(a \otimes b)=f_{1}(b \otimes a)=1$ then, the fact that $f_{1}(a \otimes a)=f_{2}(a \otimes a)=$ $f_{1}(b \otimes b)=f_{2}(b \otimes b)=1$ comes directly from relations (2) given in Theorem 3.5. To prove $f_{1}(a \otimes c)=f_{1}(b \otimes c) \in\{0,1\}$ and $f_{1}(c \otimes a)=f_{1}(c \otimes b) \in\{0,1\}$ for any $c \in X \backslash\{a, b\}$, we use the relation

$$
f_{1}(x \otimes y) f_{1}(y \otimes z)\left[f_{1}(x \otimes z)-1\right]=0 \text { for any } x, y, z \in X .
$$

Proposition 3.8. Let $\mathbb{K}$ be a field of characteristic $0, X$ be a countable alphabet and $\square$ a weak shuffle product on $\mathbb{K}\langle X\rangle$. We denote by $T$ the set $T=\left\{a \in X, f_{1}(a \otimes a) \in\right.$ $\mathbb{K} \backslash\{0,1\}\}$. We assume $T \neq \emptyset$; so $T$ is a singleton $\{a\}$. Let $\square^{\prime}$ be the weak shuffle product defined by

- $f_{1}^{\prime}(u \otimes v)=f_{1}(u \otimes u)$ for any $u \otimes v \in X \otimes X \backslash\{a \otimes a\}$,

- $f_{1}^{\prime}(a \otimes a)=1$ and $f_{2}^{\prime}(a \otimes a)=1$.

Then, there exists an algebra isomorphism between $(\mathbb{K}\langle X\rangle, \square)$ and $\left(\mathbb{K}\langle X\rangle, \square^{\prime}\right)$.

Proof. Thanks to Corollary 3.7, we know that the weak shuffle $\square$ does not depend on the value of $f_{1}(a \otimes a)$. We define $\psi:(\mathbb{K}\langle X\rangle, \square) \rightarrow\left(\mathbb{K}\langle X\rangle, \square^{\prime}\right)$ by:

$$
\psi(w)= \begin{cases}w & \text { if } w \notin a X^{*}, \\ \frac{1}{n !} w & \text { if } w=\underbrace{a \ldots a}_{n \text { times }} w_{1} \text { with } w_{1} \notin a X^{*} .\end{cases}
$$

Since $f_{1}(a \otimes b)=1$ and $f_{1}(b \otimes a)=0$ for any $b \in X \backslash\{a\}$, the linear map $\psi$ is an algebra morphism. It is trivially an isomorphism.

Proposition 3.9. Let $\mathbb{K}$ be a field of characteristic 0 , let $X$ be an alphabet of cardinality 2 or 3 and let $\square$ be a weak shuffle product on $\mathbb{K}\langle X\rangle$. Let $\square^{\prime}$ be the weak shuffle product defined by

- $f_{1}^{\prime}(a \otimes b)=1$ and $f_{1}^{\prime}(b \otimes a)=0$ for any $(a \otimes b) \in X \otimes X$ such that $a \neq b$ and $f_{1}(a \otimes b) \notin\{0,1\}$. 
- $f_{1}^{\prime}(a \otimes b)=f_{1}(a \otimes b)$ for any $(a \otimes b) \in X \otimes X$ such that $a \neq b$ and $f_{1}(a \otimes b) \in\{0,1\}$.

- $f_{i}^{\prime}(a \otimes a)=f_{i}(a \otimes a)$ for any $a \in X$ and any $i \in\{1,2\}$.

Then, there exists an algebra isomorphism between $(\mathbb{K}\langle X\rangle, \square)$ and $\left(\mathbb{K}\langle X\rangle, \square^{\prime}\right)$.

Proof. If $X=\{a, b\}$ then there is an one-parameter family of weak shuffle products $\square$ such that $f_{1}(a \otimes b) \notin\{0,1\}$. They are defined by $f_{1}(a \otimes b)=k \in \mathbb{K} \backslash\{0,1\}$ and $f_{1}(b \otimes a)=f_{1}(a \otimes a)=f_{2}(a \otimes a)=f_{1}(b \otimes b)=f_{2}(b \otimes b)=0$. We define $\square^{\prime}$ by changing $k$ in 1 . The map $\varphi$ defined by

$$
\varphi(w)= \begin{cases}\frac{1}{k^{n}} w & \text { if } w=\underbrace{a \ldots a}_{n \text { times }} w^{\prime} \text { with } w^{\prime} \in b X^{*}, \\ w & \text { else, }\end{cases}
$$

is an algebra isomorphism between $(\mathbb{K}\langle X\rangle, \square)$ and $\left(\mathbb{K}\langle X\rangle, \square^{\prime}\right)$

Let us now consider the case $X=\{a, b, c\}$. Without loss of generality we assume $f_{1}(a \otimes b)=k \in \mathbb{K} \backslash\{0,1\}$. The charactarisation of weak shuffle products given in Theorem 3.5 leads to the following relations:

- $f_{1}(b \otimes a)=f_{1}(a \otimes a)=f_{2}(a \otimes a)=f_{1}(b \otimes b)=f_{2}(b \otimes b)=0$,

- $f_{1}(a \otimes c) f_{1}(c \otimes a)=0$,

- $f_{1}(b \otimes c) f_{1}(c \otimes b)=0$,

- $f_{1}(a \otimes c) f_{1}(c \otimes b)=0$,

- $f_{1}(b \otimes c) f_{1}(c \otimes a)=0$,

- $f_{1}(u \otimes v) f_{1}(v \otimes w)\left[f_{1}(u \otimes w)-1\right]=0$ where $\{u, v, w\}=X$.

Thus, the weak shuffle product $\square$ is one of the following:

(1) $f_{1}(a \otimes c)=f_{1}(b \otimes c)=f_{1}(c \otimes a)=f_{1}(c \otimes b)=0$ and $f_{1}(c \otimes c)=f_{2}(c \otimes c) \in$ $\{0,1\}$.

(2) $f_{1}(a \otimes c)=1, f_{1}(b \otimes c)=p \in \mathbb{K}^{*}$ and $f_{1}(c \otimes a)=f_{1}(c \otimes b)=f_{1}(c \otimes c)=$ $f_{2}(c \otimes c)=0$

(3) $f_{1}(a \otimes c)=1, f_{1}(b \otimes c)=1, f_{1}(c \otimes a)=f_{1}(c \otimes b)=0$ and $f_{1}(c \otimes c)=$ $f_{2}(c \otimes c)=1$

(4) $f_{1}(a \otimes c)=f_{1}(b \otimes c)=0, f_{1}(c \otimes a)=p \in \mathbb{K}^{*}, f_{1}(c \otimes b)=1$ and $f_{1}(c \otimes c)=$ $f_{2}(c \otimes c)=0$,

(5) $f_{1}(a \otimes c)=f_{1}(b \otimes c)=0, f_{1}(c \otimes a)=1, f_{1}(c \otimes b)=1$ and $f_{1}(c \otimes c)=$ $f_{2}(c \otimes c)=1$,

(6) $f_{1}(a \otimes c)=f_{1}(b \otimes c)=0, f_{1}(c \otimes a)=1, f_{1}(c \otimes b)=1$ and $f_{1}(c \otimes c)=$ $1-f_{2}(c \otimes c)$,

(7) $f_{1}(a \otimes c)=f_{1}(b \otimes c)=f_{1}(c \otimes a)=0, f_{1}(c \otimes b)=p \in \mathbb{K}^{*}$ and $f_{1}(c \otimes c)=$ $f_{2}(c \otimes c)=0$, 
(8) $f_{1}(a \otimes c)=f_{1}(b \otimes c)=f_{1}(c \otimes a)=0, f_{1}(c \otimes b)=1$ and $f_{1}(c \otimes c)=f_{2}(c \otimes c)=$ 1 ,

(9) $f_{1}(a \otimes c)=p \in \mathbb{K}^{*}, f_{1}(b \otimes c)=f_{1}(c \otimes a)=f_{1}(c \otimes b)=0$ and $f_{1}(c \otimes c)=$ $f_{2}(c \otimes c)=0$

(10) $f_{1}(a \otimes c)=1, f_{1}(b \otimes c)=f_{1}(c \otimes a)=f_{1}(c \otimes b)=0$ and $f_{1}(c \otimes c)=f_{2}(c \otimes c)=$ 1.

We define $\square^{\prime}$ by $f_{1}^{\prime}(a \otimes b)=1$ and $f_{1}^{\prime}(u \otimes v)=f_{1}(u \otimes v)$ if $u \otimes v \neq a \otimes b$. Let $\varphi_{1}$ and $\varphi_{2}$ be the maps defined by: for any word $w$,

$$
\varphi_{1}(w)= \begin{cases}\frac{1}{k^{n}} w & \text { if } w=\underbrace{a \ldots a}_{n \text { times }} w^{\prime} \text { with } w^{\prime} \in b X^{*}, \\ w & \text { else, }\end{cases}
$$

and

$\varphi_{2}(w)= \begin{cases}\frac{1}{k^{n_{1}+\cdots+n_{s}}} w & \text { if } w=\underbrace{c \ldots c}_{q_{1} \text { times }} \underbrace{a \ldots a}_{n_{1} \text { times }} \underbrace{c \ldots c}_{q_{2} \text { times }} \cdots \underbrace{a \ldots a}_{q_{s} \text { times } n_{s} \text { times } q_{s+1} \ldots c} \underbrace{c \ldots c}_{\text {times }} w^{\prime} \text { with } w^{\prime} \in b X^{*} \\ & \text { and }\left(q_{1}, \ldots, q_{s+1}\right) \in \mathbb{N}^{s+1}, \\ & \text { else. }\end{cases}$

From case 1 to case 3 and from case 9 to case 10 the map $\varphi_{1}$ is an algebra isomorphism between $(\mathbb{K}\langle X\rangle, \square)$ and $\left(\mathbb{K}\langle X\rangle, \square^{\prime}\right)$. From case 4 to case 8 the map $\varphi_{2}$ is an algebra isomorphism between $(\mathbb{K}\langle X\rangle, \square)$ and $\left(\mathbb{K}\langle X\rangle, \square^{\prime}\right)$.

If maps $f_{1}^{\prime}$ and $f_{2}^{\prime}$ do not take their values in $\{0,1\}$ we apply the previous process once again to $\square^{\prime}$. And then, we find a weak shuffle product $\square^{\prime \prime}$ such that $f_{1}{ }^{\prime \prime}(u \otimes v), f_{2}{ }^{\prime \prime}(u \otimes v) \in\{0,1\}$ for any $(u \otimes v) \in X \otimes X$.

Conjecture 3.10. Proposition 3.9 is still true for any countable alphabet.

Remark 3.11. If $X$ is an alphabet such that $\{a, b, c, d\} \subset X$ and $f_{1}(a \otimes b) \notin\{0,1\}$ then relations

(1) $f_{1}(a \otimes x) f_{1}(x \otimes a)=0$,

(2) $f_{1}(b \otimes x) f_{1}(x \otimes b)=0$

(3) $f_{1}(a \otimes x) f_{1}(x \otimes b)=0$,

(4) $f_{1}(b \otimes x) f_{1}(x \otimes a)=0$,

are still satisfied for any letter $x \in X$. However, if $x, y \in X \backslash\{a, b\}$, even if they satisfy relations given in Theorem 3.5, it is hard to anticipate the part of $x$ facing $y$. 
3.2. Weak shuffle products on $\mathbb{K}\langle\{a, b\}\rangle$. Let $X=\{a, b\}$ be an alphabet of cardinality 2. By using the characterisation given in Theorem 3.5, there are 10 families of weak shuffle products defined on $\mathbb{K}\langle X\rangle$. Let $C$ be the 6 -tuple $C=$ $\left(f_{1}(a \otimes b), f_{1}(b \otimes a), f_{1}(a \otimes a), f_{2}(a \otimes a), f_{1}(b \otimes b), f_{2}(b \otimes b)\right)$. If $k \in \mathbb{K}^{*}$ and $\alpha \in \mathbb{K}$ then $C$ is one of the following 6 -tuples

$$
\begin{aligned}
& C_{1}=(0,0,0,0,0,0), \quad C_{2}=(k, 0,0,0,0,0), \quad C_{3}=(1,0,1,1,0,0), \\
& C_{4}=(1,0,0,0,1,1), \quad C_{5}=(0,0,1,1,0,0), \quad C_{6}=(0,0,1,1,1,1) \text {, } \\
& C_{7}=(1,0, \alpha, 1-\alpha, 0,0), \quad C_{8}=(1,0, \alpha, 1-\alpha, 1,1), \quad C_{9}=(1,0,1,1,1,1), \\
& C_{10}=(1,1,1,1,1,1) \text {. }
\end{aligned}
$$

For any $n \in \llbracket 1,10 \rrbracket$, we denote by $\square$ the weak shuffle product associated to $C_{n}$. The concatenation of two words $u$ and $v$ is denoted by $u v$. The empty word is denoted by 1 .

Case $n=2$ : Thanks to Proposition 3.9, for any $k \in \mathbb{K}^{*}$ the weak shuffle product defined by $C_{2}$ is isomorphic to the case $(1,0,0,0,0,0)$. Let $u$ and $v$ be two non-empty words. Then

$$
u \square v= \begin{cases}k^{n} u v & \text { if }(u=\underbrace{a \ldots a}_{n \text { times }} \text { and } v=b w \text { with } w \in X^{*}) \\ k^{n} v u & \text { if }\left(v=a \ldots a \text { and } u=b w \text { with } w \in X^{*}\right) \\ 0 & \text { else. }\end{cases}
$$

Cases $n=3$ and $n=7$ : Thanks to Proposition 3.8 the weak shuffle products defines by $C_{3}$ and $C_{7}$ are isomorphic. Let $u$ and $v$ be two non-empty words. Then

$$
u \underset{3}{\square} v= \begin{cases}u v & \text { if }\left(u=a \ldots a \text { and } v=b w \text { with } w \in X^{*}\right) \\
v u & \text { if }\left(v=a \ldots a \text { and } u=b w \text { with } w \in X^{*}\right), \\
\left(\begin{array}{c}
k+l \\
k
\end{array}\right) \underbrace{a \ldots a}_{k+l \text { times }} w & \text { if }(u=\underbrace{a \ldots a}_{k \text { times }} \text { and } v=\underbrace{a \ldots a}_{l \text { times }} w \text { with } w \in b X^{*} \cup\{1\}) \\
& \text { or }(v=\underbrace{a \ldots a}_{k \text { times }} \text { and } u=\underbrace{a \ldots a}_{l \text { times }} w \text { with } w \in b X^{*} \cup\{1\}), \\
& \text { else, }\end{cases}
$$




$$
\text { and } u \square v= \begin{cases}u v & \text { if }\left(u=a \ldots a \text { and } v=b w \text { with } w \in X^{*}\right) \\ v u & \text { if }\left(v=a \ldots a \text { and } u=b w \text { with } w \in X^{*}\right), \\ \underbrace{a \ldots a}_{k+l \text { times }} w & \text { if }(u=\underbrace{a \ldots a}_{k \text { times }} \text { and } v=\underbrace{a \ldots a}_{l \text { times }} w \text { with } w \in b X^{*} \cup\{1\}) \\ & \text { or }(v=\underbrace{a \ldots a}_{k \text { times }} \text { and } u=\underbrace{a \ldots a}_{l \text { times }} w \text { with } w \in b X^{*} \cup\{1\}), \\ 0 & \text { else. }\end{cases}
$$

Case $n=5$ : Let $u$ and $v$ be two non-empty words. Then

$$
u \square v= \begin{cases}\left(\begin{array}{c}
k+l-1 \\
k
\end{array}\right) \underbrace{a \ldots a}_{k+l \text { times }} w & \text { if }(u=\underbrace{a \ldots a}_{k \text { times }} \text { and } v=\underbrace{a \ldots a}_{l \text { times }} w \text { with } w \in b X^{*}) \\
\left(\begin{array}{c}
k+l \\
k
\end{array}\right) \underbrace{a \ldots a}_{k+l \text { times }} & \text { or }(v=\underbrace{a \ldots a}_{k \text { times }} \text { and } u=\underbrace{a \ldots a}_{l \text { times }} w \text { with } w \in b X^{*}), \\
0 & \text { if } u=\underbrace{a \ldots a}_{k \text { times }} \text { and } v=\underbrace{a \ldots a}_{l \text { times }},\end{cases}
$$

Case $n=6$ : Let $u$ and $v$ be two non-empty words. Then

$$
u \square v= \begin{cases}\left(\begin{array}{c}
k+l-1 \\
k
\end{array}\right) \underbrace{a \ldots a}_{k+l \text { times }} w & \text { if }(u=\underbrace{a \ldots a}_{k \text { times }} \text { and } v=\underbrace{a \ldots a}_{l \text { times }} w \text { with } w \in b X^{*}) \\
\left(\begin{array}{c}
k+l \\
k
\end{array}\right) \underbrace{a \ldots a}_{k+l \text { times }} & \text { or }(v=\underbrace{a \ldots a}_{k \text { times }} \text { and } u=\underbrace{a \ldots a}_{l \text { times }} w \text { with } w \in b X^{*}), \\
\left(\begin{array}{c}
k+l-1 \\
k
\end{array}\right) \underbrace{b \ldots b}_{k+l \text { times }} w & \text { if }(u=\underbrace{b \ldots b}_{k \text { times }} \text { and } v=\underbrace{b \ldots b}_{l \text { times }} w \text { with } w \in a X^{*}) \\
\left(\begin{array}{c}
k+l \\
k
\end{array}\right) \underbrace{b \ldots b}_{k+l \text { times }} & \text { or }(v=\underbrace{b \ldots b}_{k \text { times }} \text { and } u=\underbrace{b \ldots b}_{l \text { times }} w \text { with } w \in a X^{*}), \\
0 & \text { if } u=\underbrace{b \ldots b}_{k \text { times }} \text { and } v=\underbrace{b \ldots b}_{l \text { times }},\end{cases}
$$

Case $n=4$ : First, it is natural to ask whether or not this case is isomorphic to the case with $n=3$ ? In fact, not. A counter-example is given by the elements $u$ of degree 2 such that $u^{2}=0$. Indeed,

(1) with the case $n=4$, if $u=\lambda a a+\mu b b+\sigma a b+\tau b a$ then

$$
\begin{aligned}
u^{2} & =6 \mu^{2} b b b b+2 \tau^{2} b a b a+2 \lambda \mu a a b b+2 \lambda \tau a a b a+6 \mu \sigma a b b b \\
& +2 \mu \tau(b a b b+b b a b+b b b a)+2 \sigma \tau(a b a b+a b b a) .
\end{aligned}
$$


So $u^{2}=0 \Longleftrightarrow \mu=\tau=0$ and $\{u \in \mathbb{K}\langle\{a, b\}\rangle$, length $(u)=$ 2 and $\left.u^{2}=0\right\}=\operatorname{Span}(a a, a b)$.

(2) with the case $n=3$, if $u=\lambda a a+\mu b b+\sigma a b+\tau b a$ then

$$
u^{2}=6 \lambda^{2} a a a a+2 \lambda \mu a a b b+6 \lambda \sigma a a a b+2 \lambda \tau a a b a .
$$

So $u^{2}=0 \Longleftrightarrow \lambda=0$ and $\left\{u \in \mathbb{K}\langle\{a, b\}\rangle\right.$, length $(u)=2$ and $u^{2}=$ $0\}=\operatorname{Span}(b b, a b, b a)$.

Let $u$ and $v$ be two non-empty words. Then

(1) If $u=\underbrace{a \ldots a}_{m \text { times }} u^{\prime}$ and $u^{\prime}, v \in b X^{*} \cup\{1\}$ then

$$
u \square v=v \underset{4}{\square} u=\underbrace{a \ldots a}_{m \text { times }}\left(u^{\prime} \square v\right) .
$$

(2) If $u=\underbrace{b \ldots b}_{m_{1} \text { times }} u^{\prime}, v=\underbrace{b \ldots b}_{m_{2} \text { times }} v^{\prime}$ and $u^{\prime}, v^{\prime} \in a X^{*} \cup\{1\}$ then

$$
\begin{aligned}
u \square v= & \sum_{k=0}^{m_{2}-1}\left(\begin{array}{c}
m_{1}+k-1 \\
k
\end{array}\right) \underbrace{b \ldots b}_{m_{1}+k \text { times }}(u^{\prime} \underbrace{b \ldots b}_{m_{2}-k \text { times }} w^{\prime}) \\
& +\sum_{k=0}^{m_{1}-1}\left(\begin{array}{c}
m_{2}+k-1 \\
k
\end{array}\right) \underbrace{b \ldots b}_{m_{2}+k \text { times }}(\underbrace{b \ldots b}_{m_{1}-k \text { times }} u^{\prime} \square v^{\prime}) \\
= & v \square u
\end{aligned}
$$

(3) If $u, v \in a X^{*}$ then $u \square v=v \square u=0$.

Cases $n=8$ and $n=9$ : We recall that the case $n=8$ does not depend on $\alpha \in \mathbb{K}$. Thanks to Proposition 3.8 the weak shuffle products defined by $C_{8}$ and $C_{9}$ are isomorphic. Let $u$ and $v$ be two non-empty words. Then

(1) If $u=\underbrace{a \ldots a}_{m \text { times }} u^{\prime}$ and $u^{\prime}, v \in b X^{*} \cup\{1\}$ then

$$
u \underset{9}{\square} v=v \underset{9}{\square} u=\underbrace{a \ldots a}_{m \text { times }}\left(u^{\prime} \square v\right)=u \underset{8}{\square} v=v \underset{8}{\square} u .
$$


(2) If $u=\underbrace{b \ldots b}_{m_{1} \text { times }} u^{\prime}, v=\underbrace{b \ldots b}_{m_{2} \text { times }} v^{\prime}$ and $u^{\prime}, v^{\prime} \in a X^{*} \cup\{1\}$ then

$$
\begin{aligned}
u \square v & =\sum_{k=0}^{m_{2}-1}\left(\begin{array}{c}
m_{1}+k-1 \\
k
\end{array}\right) \underbrace{b \ldots b}_{m_{1}+k \text { times }}(u_{9}^{\prime} \underbrace{b \ldots b}_{m_{2}-k \text { times }} v^{\prime}) \\
& +\sum_{k=0}^{m_{1}-1}\left(\begin{array}{c}
m_{2}+k-1 \\
k
\end{array}\right) \underbrace{b \ldots b}_{m_{2}+k \text { times }}(\underbrace{b \ldots b}_{m_{1}-k \text { times }} u^{\prime} v_{9}^{\prime} v^{\prime}) \\
= & v \square u=u \square v=v \square u .
\end{aligned}
$$

(3) If $u=\underbrace{a \ldots a}_{k \text { times }} u^{\prime}, v=\underbrace{a \ldots a}_{l \text { times }} v^{\prime}$ and $u^{\prime}, v^{\prime} \in b X^{*} \cup\{1\}$ then

$$
u \underset{9}{\square} v=v \underset{9}{\square} u=\left(\begin{array}{c}
k+l \\
k
\end{array}\right) \underbrace{a \ldots a}_{k+l \text { times }}\left(u_{9}^{\prime} v^{\prime}\right),
$$

and

$$
u \underset{8}{\square} v=v \underset{8}{\square} u=\underbrace{a \ldots a}_{k+l \text { times }}\left(u_{8}^{\prime} \square v^{\prime}\right) .
$$

From the previous calculations, we have the following consequence:

Corollary 3.12. Let $v$ and $w$ be two words. Then $\underset{9}{\square} w \neq 0$.

Remark 3.13. For cases $n \in\{4,8,9\}$, since $f_{1}(a \otimes b)=1$ and $f_{1}(b \otimes a)=0$, the calculation of $u \square v$ where $u=\underbrace{b \ldots b}_{m_{1} \text { times }} u^{\prime}, v=\underbrace{b \ldots b}_{m_{2} \text { times }} v^{\prime}$ and $u^{\prime}, v^{\prime} \in$ $a X^{*} \cup\{1\}$ does not depend on the values of $f_{1}(a \otimes a)$ nor $f_{2}(a \otimes a)$. We give the value of $u \square v(=u \square v=u \square v)$ for some example couple $(u, v) \in\left(b X^{*}\right)^{2}$. For some examples of pairs $(x, p) \in X \times \mathbb{N}^{*}$, to lighten the notation, we write $x^{p}$ instead of $\underbrace{x \ldots x}_{p \text { times }}$.

Let $(m, s, p, r)$ be a quadruple of positive integers. Then:

$$
b^{m} a_{4}^{s} b^{p} a^{r}=\sum_{k=0}^{p-1}\left(\begin{array}{c}
m+k-1 \\
k
\end{array}\right) b^{m+k} a^{s} b^{p-k} a^{r}+\sum_{k=0}^{m-1}\left(\begin{array}{c}
p+k-1 \\
k
\end{array}\right) b^{p+k} a^{r} b^{m-k} a^{s} .
$$

Let $(m, s, p, r, t)$ be a quintuple of positive integers such that $m \geq 2$. Then:

$$
\begin{aligned}
b^{m} a_{4}^{s} b^{p} a^{r} b^{t}= & \sum_{k=0}^{p-1}\left(\begin{array}{c}
m+k-1 \\
k
\end{array}\right) b^{m+k} a^{s} b^{p-k} a^{r} b^{t}+\sum_{k=0}^{t}\left(\begin{array}{c}
m+k-1 \\
k
\end{array}\right) b^{p} a^{r} b^{m+k} a^{s} b^{t-k} \\
& +\sum_{\substack{f+g=m \\
f \in \mathbb{N}^{*} \\
g \in \mathbb{N}^{*}}} \sum_{k=0}^{t}\left(\begin{array}{c}
f+p-1 \\
f
\end{array}\right)\left(\begin{array}{c}
g+k-1 \\
k
\end{array}\right) b^{p+f} a^{r} b^{g+k} a^{s} b^{t-k}
\end{aligned}
$$


Proposition 3.14. Let $\square$ be the weak shuffle product defined by $C_{9}$. Let $p$ be a positive integer and $n \in\{1,2,3\}$. We denote by $K_{(n, p)}$ the set

$$
K_{(n, p)}=\left\{u=\sum_{\substack{w \in X^{*} \\ \text { length }(w)=n}} \lambda_{w} w, u^{p}=0\right\} .
$$

Then, $K_{(n, p)}=\{0\}$.

Proof. We equip $X^{*}$ with the lexicographic order. For any words $v$ and $w$ we denote by $\max (v \square w)$ the greatest word of length $l=\operatorname{length}(v)+\operatorname{length}(w)$ which appears in $v \square w$ for the lexicographic order.

$$
\begin{aligned}
& \text { If } u=\sum_{\substack{w \in X^{*} \\
\text { length }(w)=n}} \lambda_{w} w \text { then }
\end{aligned}
$$

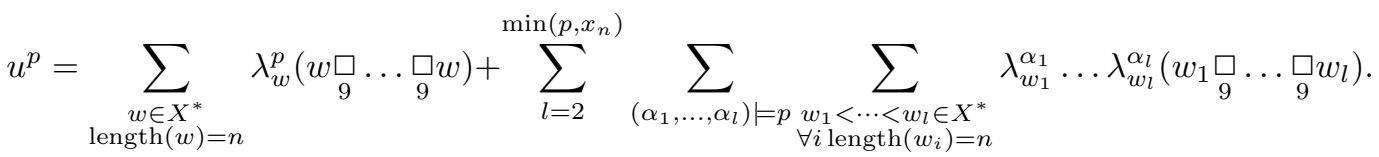

(1) If $n=1$ then the result is trivial.

(2) If $n=2$ then

$$
a a^{p}=\frac{(2 p) !}{2^{p}} \underbrace{a \ldots a}_{2 p \text { times }}, \quad a b^{p}=(p !)^{2} \underbrace{a \ldots a}_{p \text { times } p \text { times }} \underbrace{b \ldots b}, \quad b a^{p}=p ! \underbrace{b a \ldots b a}_{p \text { times }}, \quad b b^{p}=\frac{(2 p) !}{2^{p}} \underbrace{b \ldots b}_{2 p \text { times }},
$$

and

$$
\max \left(a a^{k} \underset{9}{\square} a b^{l}{ }_{9} b a^{m}{ }_{9}^{\square} b b^{n}\right)=\underbrace{a \ldots a}_{\substack{2 k+l \\
\text { times }}} \underbrace{b \ldots b}_{\begin{array}{c}
2 n+l \\
\text { times }
\end{array}} \underbrace{b a \ldots b}_{\begin{array}{c}
m \\
\text { times }
\end{array}} .
$$

Thus $\lambda_{a a}=\lambda_{b b}=\lambda_{b a}=\lambda_{a b}=0$.

(3) If $n=3$ then

$$
\begin{array}{ll}
w_{1}=a a a^{p}=\frac{(3 p) !}{(3 !)^{p}} \underbrace{a \ldots a}_{3 p \text { times }}, & w_{2}=a a b^{p}=\frac{(2 p) ! p !}{2^{p}} \underbrace{a \ldots a}_{2 p \text { times }} \underbrace{b \ldots b}_{p \text { times }}, \\
w_{3}=a b a^{p}=(p !)^{2} \underbrace{a \ldots a}_{p \text { times }} \underbrace{b a \ldots b a}_{p \text { times }}, & w_{4}=a b b^{p}=\frac{(2 p) ! p !}{2^{p}} \underbrace{a \ldots a}_{p \text { times } 2 p \text { times }} \underbrace{b \ldots b}_{p \text { times }}, \\
w_{5}=b a a^{p}=p ! \underbrace{b a a \ldots b a a}, & w_{6}=b b b^{p}=\frac{(3 p) !}{(3 !)^{p}} \underbrace{b \ldots b}_{3 p \text { times }} .
\end{array}
$$

For $b a b^{p}$ and $b b a^{p}$, there are several terms in the result. For $b a b^{p}$ we will use $w_{7}=\underbrace{b a b \ldots b a b}_{p \text { times }}$ and, for $b b a^{n}$ we will use $w_{8}=\underbrace{b \ldots b}_{p \text { times }} \underbrace{b a \ldots b a}_{p \text { times }}$. In fact, for the lexicographic order, we use the maximal term obtained in each product. For any $i$ we determine how build $w_{i}$ by doing the weak shuffle of $p$ words 
of length 3. We get $\lambda_{a a a}=\lambda_{b b b}=\lambda_{a b a}=\lambda_{b a a}=\lambda_{a a b}=\lambda_{a b b}=\lambda_{b a b}=$ $\lambda_{b b a}=0$.

Conjecture 3.15. Let $\square_{9}$ be the weak shuffle product defined by $C_{9}$. For any positive integers $p$ and $n$, we have $K_{(n, p)}=\{0\}$.

Remarks 3.16. (1) By induction we can express $\max \underset{9}{\square} v)$ for any words $u$ and $v$.

Case $w_{1}$ and $w_{2}$ are in $a X^{*}$ : There exist $\alpha, \beta \in \mathbb{N}^{*}$ and $w_{1}^{\prime}, w_{2}^{\prime} \in b X^{*} \cup$

$\{1\}$ such that $w_{1}=\underbrace{a \ldots a}_{\alpha \text { times }} w_{1}^{\prime}$ and $w_{2}=\underbrace{a \ldots a}_{\beta \text { times }} w_{2}^{\prime}$. Then,

$$
\max \left(w_{1} \underset{9}{\square} w_{2}\right)=\underbrace{a \ldots a}_{\alpha+\beta \text { times }} \max \left(w_{1}^{\prime} \square w_{2}^{\prime}\right) .
$$

Case $w_{1} \in a X^{*}$ and $w_{2} \in b X^{*}$ : There exist $\alpha \in \mathbb{N}^{*}$ and $w_{1}^{\prime} \in b X^{*} \cup\{1\}$ such that $w_{1}=\underbrace{a \ldots a}_{\alpha \text { times }} w_{1}^{\prime}$. Then,

$$
\max \left(w_{1} \square w_{2}\right)=\underbrace{a \ldots a}_{\alpha \text { times }} \max \left(w_{1}^{\prime} \square w_{2}\right) .
$$

Case $w_{1}$ and $w_{2}$ are in $b X^{*}:$ There exist $\alpha, \beta \in \mathbb{N}^{*}, p, q \in \mathbb{N}$ (they are not necessarily different from 0 ) and $w_{1}^{\prime}, w_{2}^{\prime} \in b X^{*} \cup\{1\}$ such that $w_{1}=\underbrace{b \ldots b}_{\alpha \text { times } p \text { times }} \underbrace{a \ldots a}_{1} w_{1}^{\prime}$ and $w_{2}=\underbrace{b \ldots b}_{\beta \text { times }} \underbrace{a \ldots a}_{\text {times }} w_{2}^{\prime}$. Thus,

- If $0<q<p$ then

$$
\max \left(w_{1} \square w_{2}\right)=\underbrace{b \ldots b}_{\alpha+\beta-1 \text { times }} \underbrace{a \ldots a}_{q \text { times }} \max (b \underbrace{a \ldots a}_{p \text { times }} w_{1}^{\prime} \square w_{2}^{\prime}) .
$$

- If $0<p<q$ then

$$
\max \left(w_{1} \square w_{9}\right)=\underbrace{b \ldots b}_{\alpha+\beta-1 \text { times } p \text { times }} \underbrace{a \ldots a}_{9} \max (w_{1}^{\prime} \square b \underbrace{a \ldots a}_{q \text { times }} w_{2}^{\prime}) .
$$

- If $0<p$ and $p=q$ then $\max \left(w_{1} \underset{9}{\square} w_{2}\right)=\max \left(\tilde{w}_{1}, \tilde{w}_{2}\right)$ where

$$
\tilde{w}_{1}=\underbrace{b \ldots b}_{\alpha+\beta-1 \text { times }} \underbrace{a \ldots a}_{\text {times }} \max (b \underbrace{a \ldots a}_{p \text { times }} w_{1}^{\prime} \square w_{2}^{\prime})
$$

and

$$
\tilde{w}_{2}=\underbrace{b \ldots b}_{\alpha+\beta-1 \text { times }} \underbrace{a \ldots a}_{p \text { times }} \max (w_{1}^{\prime} \square b \underbrace{a \ldots a}_{q \text { times }} w_{2}^{\prime})) .
$$


- If $p=0$ (respectively $q=0$ ) then $w_{1}=\underbrace{b \ldots b}_{\alpha \text { times }}$ (respectively $w_{2}=\underbrace{b \ldots b}_{\beta \text { times }})$ and

$\max \left(\left(w_{1} \underset{9}{\square} w_{2}\right)\right)=w_{1} w_{2}$ (respectively $\left.\max \left(\left(w_{1} \underset{9}{\square} w_{2}\right)\right)=w_{2} w_{1}\right)$.

For instance,

$$
\begin{aligned}
& \max (a b \underset{9}{\square} a b a a)=a a \max (\underset{9}{\square} b a a)=a a b b a a, \\
& \max (b b a \underset{9}{\square} b a a)=b b a b a a, \\
& \max (b b b a a a b b a \underset{9}{\square} b b a a b b b a)=b b b b a a \max (b a a a b b a \underset{9}{\square} b b b a)=b b b b a a b b b a b a a a b b a .
\end{aligned}
$$

(2) For $p=2$ Conjecture 3.15 is implied by the statement "Let $n$ be a positive integer, let $w_{1}, w_{2}$ and $w$ be three non-empty words of length $n$ such that $w_{1} \leq w_{2} \leq w$ and $w_{1}<w$. Then $\max \left(w_{1} \square w_{2}\right)<\max (w \underset{9}{\square} w) "$ We attend a reasoning by induction but there are some obstructions. Indeed, it leads us to compare $\max \left(u_{1} \square u_{2}\right)$ and $\max \left(u_{3} \square{ }_{9}^{\square} u_{4}\right)$ where $u_{1} \leq u_{3}$, $u_{2} \leq u_{4}, \operatorname{length}\left(u_{1}\right)=\operatorname{length}\left(u_{3}\right), \operatorname{length}\left(u_{2}\right)=\operatorname{length}\left(u_{4}\right)$ and $\left(u_{1}, u_{2}\right) \neq$ $\left(u_{3}, u_{4}\right)$. Then, it leads us to determine if $\max \left(v_{1} \square v_{2}\right)>\max \left(v_{3} \underset{9}{\square} v_{4}\right)$ or $\max \left(v_{1} \underset{9}{\square} v_{2}\right)<\max \left(v_{3} \underset{9}{\square} v_{4}\right)$ where $v_{1}<v_{3}, v_{2}>v_{4}$. If we consider $v_{1}=a$, $v_{2}=b b, v_{3}=a b$ and $v_{4}=b$, then we get $\max \left(v_{1} \underset{9}{\square} v_{2}\right)=a b b=\max \left(v_{3} \underset{9}{\square} v_{4}\right)$.

By using computation programs realised with Maxima, (c.f. Section 7 ) we get:

Lemma 3.17. Let $n$ be a positive integer smaller than or equal to 7 . Then $K_{n, 2}=$ $\{0\}$.

Proposition 3.18. Let $X$ be the alphabet $\{a, b\}$ and $\mathcal{S}$ be the set defined by $\mathcal{S}=\left\{C_{1} \ldots C_{10}\right\}$ equipped with the relation $\equiv$ such that: for any $A$ and $B$ in $\mathcal{S}$, $A \equiv B$ if and only if there exists an homogenous isomorphism between $\left(\mathbb{K}\langle X\rangle, \square_{A}\right)$ and $\left(\mathbb{K}\langle X\rangle, \square_{B}\right)$ where $\square_{A}$ (respectively $\square_{B}$ ) is the shuffle product associated to $A$ (respectively $B)$. Let $n$ be the number of isomorphic classes.

Then $n \in\{7,8\}$.

3.3. Weak shuffle products on $\mathbb{K}\langle\{a, b, c\}\rangle$. Let $X=\{a, b, c\}$ be an alphabet of cardinality 3. Let $C$ be the 12-tuple $C=\left(f_{1}(a \otimes b), f_{1}(b \otimes a), f_{1}(b \otimes c), f_{1}(c \otimes\right.$ $\left.b), f_{1}(a \otimes c), f_{1}(c \otimes a) f_{1}(a \otimes a), f_{2}(a \otimes a), f_{1}(b \otimes b), f_{2}(b \otimes b), f_{1}(c \otimes c), f_{2}(c \otimes c)\right)$. By using Theorem 3.5, if $(k, m) \in\left(\mathbb{K}^{*}\right)^{2}$ and $\alpha \in \mathbb{K}$ then $C$ is one of the following 
tuples

$$
\begin{aligned}
& C_{1}=(0,0,0,0,0,0,0,0,0,0,0,0), \quad C_{2}=(0,0,0,0,0,0,1,1,0,0,0,0), \\
& C_{3}=(0,0,0,0,0,0,1,1,1,1,0,0), \quad C_{4}=(0,0,0,0,0,0,1,1,1,1,1,1), \\
& C_{5}=(k, 0,0,0,0,0,0,0,0,0,0,0), \quad C_{6}=(k, 0,0,0,0,0,0,0,0,0,1,1) \text {, } \\
& C_{7}=(1,0,0,0,0,0,1,1,0,0,0,0), \quad C_{8}=(1,0,0,0,0,0,0,0,1,1,0,0) \text {, } \\
& C_{9}=(1,0,0,0,0,0,1,1,1,1,0,0), \quad C_{10}=(1,0,0,0,0,0,1,1,0,0,1,1), \\
& C_{11}=(1,0,0,0,0,0,0,0,1,1,1,1), \quad C_{12}=(1,0,0,0,0,0,1,1,1,1,1,1) \text {, } \\
& C_{13}=(1,1,0,0,0,0,1,1,1,1,0,0), \quad C_{14}=(1,1,0,0,0,0,1,1,1,1,1,1) \text {, } \\
& C_{15}=(k, 0,0, m, 0,0,0,0,0,0,0,0), \quad C_{16}=(k, 0,0,1,0,0,0,0,0,0,1,1), \\
& C_{17}=(1,0,0,1,0,0,0,0,1,1,0,0), \quad C_{18}=(1,0,0,1,0,0,1,1,1,1,0,0), \\
& C_{19}=(1,0,0,1,0,0,1,1,0,0,1,1), \quad C_{20}=(1,0,0,1,0,0,1,1,1,1,1,1), \\
& C_{21}=(k, 0,0,0, m, 0,0,0,0,0,0,0), \quad C_{22}=(1,0,0,0,1,0,1,1,0,0,0,0), \\
& C_{23}=(1,0,0,0,1,0, \alpha, 1-\alpha, 0,0,0,0), \quad C_{24}=(k, 0,0,0,1,0,0,0,0,0,1,1), \\
& C_{25}=(1,0,0,0,1,0,1,1,1,1,0,0), \quad C_{26}=(1,0,0,0,1,0, \alpha, 1-\alpha, 1,1,0,0) \text {, } \\
& C_{27}=(1,0,0,0,1,0,0,0,1,1,1,1), \quad C_{28}=(1,0,0,0,1,0,1,1,1,1,1,1), \\
& C_{29}=(1,0,0,0,1,0, \alpha, 1-\alpha, 1,1,1,1), \quad C_{30}=(k, 0, m, 0,1,0,0,0,0,0,0,0), \\
& C_{31}=(1,0, k, 0,1,0,1,1,0,0,0,0), \quad C_{32}=(1,0,1,0,1,0,0,0,1,1,0,0), \\
& C_{33}=(k, 0,1,0,1,0,0,0,0,0,1,1), \quad C_{34}=(1,0,1,0,1,0,1,1,1,1,0,0), \\
& C_{35}=(1,0,1,0,1,0,1,1,0,0,1,1), \quad C_{36}=(1,0,1,0,1,0,0,0,1,1,1,1), \\
& C_{37}=(1,0,1,0,1,0,1,1,1,1,1,1), \quad C_{38}=(1,0, k, 0,1,0, \alpha, 1-\alpha, 0,0,0,0) \text {, } \\
& C_{39}=(1,0,1,0,1,0, \alpha, 1-\alpha, 1,1,0,0), \quad C_{40}=(1,0,1,0,1,0, \alpha, 1-\alpha, 0,0,1,1), \\
& C_{41}=(1,0,1,0,1,0, \alpha, 1-\alpha, 1,1,1,1), \quad C_{42}=(1,1,1,0,1,0,1,1,1,1,0,0), \\
& C_{43}=(1,1,1,0,1,0,1,1,1,1,1,1), \quad C_{44}=(1,1,0,1,0,1,1,1,1,1,0,0), \\
& C_{45}=(1,1,0,1,0,1,1,1,1,1,1,1), \quad C_{46}=(1,1,0,1,0,1,1,1,1,1, \alpha, 1-\alpha) \text {, } \\
& C_{47}=(1,1,1,1,1,1,1,1,1,1,1,1) \text {. }
\end{aligned}
$$

Proposition 3.19. Let $X$ be the alphabet $\{a, b, c\}$ and $\mathcal{S}$ be the set defined by $\mathcal{S}=\left\{C_{1} \ldots C_{47}\right\}$ equipped with the relation $\equiv$ such that: for any $A$ and $B$ in $\mathcal{S}$, $A \equiv B$ if and only if there exists an homogenous isomorphism between $\left(\mathbb{K}\langle X\rangle, \square_{A}\right)$ and $\left(\mathbb{K}\langle X\rangle, \square_{B}\right)$ where $\square_{A}$ (respectively $\square_{B}$ ) is the shuffle product associated to $A$ (respectively B). Let $n$ be the number of isomorphic classes.

Then $n \in \llbracket 33,39 \rrbracket$. 
Proof. Thanks to Proposition 3.9, in any set, it is sufficient to consider that $k=$ $m=1$. Thanks to Proposition 3.8, we can prove that cases $C_{22}$ and $C_{23}$ are isomorphic, cases $C_{25}$ and $C_{26}$ are isomorphic, cases $C_{28}$ and $C_{29}$ are isomorphic, cases $C_{31}$ and $C_{38}$ are isomorphic, cases $C_{34}$ and $C_{39}$ are isomorphic, cases $C_{35}$ and $C_{40}$ are isomorphic, cases $C_{37}$ and $C_{41}$ are isomorphic and cases $C_{45}$ and $C_{46}$ are isomorphic.

Let $K_{1}, K_{2}$ and $K_{3}$ be the sets defined by:

$$
\begin{aligned}
& \text { - } K_{1}=\left\{u=\sum_{x \in X} \lambda_{x} x, u^{2}=0\right\}, \\
& \text { - } K_{2}=\left\{u=\sum_{\substack{w \in X^{*} \\
\operatorname{length}(w)=2}} \lambda_{w} w, u^{2}=0\right\}, \\
& \text { - } K_{3}=\left\{u=\sum_{\substack{w \in X^{*} \\
\text { length }(w)=3}} \lambda_{w} w, u^{2}=0\right\} .
\end{aligned}
$$

By using $K_{1}$ and $K_{2}$, we conclude that $C_{6}, C_{7}$ and $C_{8}$ are in three different isomorphic classes, $C_{9}, C_{10}$ and $C_{11}$ are in three different isomorphic classes, $C_{16}$, $C_{17}, C_{22}$ and $C_{24}$ are in four different isomorphic classes, $C_{18}, C_{19}, C_{25}$ and $C_{27}$ are in four different isomorphic classes, $C_{15}$ and $C_{21}$ are in two different isomorphic classes, $C_{31}, C_{32}$ and $C_{33}$ are in three different isomorphic classes, $C_{34}, C_{35}$ and $C_{36}$ are in three different isomorphic classes, $C_{42}$ and $C_{44}$ are in two different isomorphic classes. With $K_{3}$, we prove that $C_{20}$ and $C_{28}$ are in two different isomorphic classes. Those sets do not enable us to conclude if there exists an isomorphism between $C_{9}$ and $C_{13}$, between $C_{12}$ and $C_{14}$, between $C_{34}$ and $C_{42}$, between $C_{36}$ and $C_{44}$, between $C_{43}$ and $C_{47}$, between $C_{45}$ and $C_{47}$.

\section{Weak shuffle algebras, dendriform algebras, quadri-algebras}

Dendriform algebras [22] and quadri-algebras [1] are algebraic structures which enables one to split the associativity. Actually, a dendriform algebra is an algebra $\mathcal{A}$ equipped with a left product $\prec$ and a right product $\succ$ making the couple $(\mathcal{A}, \prec$ $+\succ$ ) into an associative algebra and satisfying compatibilities. A quadri-algebra is obtained by splitting each product of a dendriform algebra in two products and the four new products must respect compatibilities. So, a quadri-algebra leads to two dendriform structures and the sum of the four products gives an associative product.

Those two notions have been extensively studied. For instance, Loday and Ronco give the free dendriform algebra on one generator as an algebra over binary planar trees [23]. Thanks to dendriform algebras, Foissy proves [9, Proposition 31] that 
the decorated Hopf algebra of Loday and Ronco and the decorated Hopf algebra of planar rooted trees are isomorphic. Analogue theorems of the Cartier-QuillenMilnor-Moore theorem have been proved: by Ronco [27] for dendriform algebras, by Chapoton [3] for dendriform bialgebras and by Foissy [10] for bidendriform bialgebras. The bidendriform case implies that FQSym is isomorphic to one decorated Hopf algebra of planar rooted trees.

About quadri-algebras, Aguiar and Loday [1] have determined a quadri-algebra structure on infinitesimal algebras and have focused on the free quadri-algebra on one generator. Vallette [32] has proved some conjectures given by Aguiar and Loday in [1, conjectures 4.2, 4.5 and 4.6]. Foissy has presented the free quadri-algebra on one generator as a sub-object of FQSym [11].

In this section, we recall the dendriform algebra structure and the quadri-algebra structure underlying the classical shuffle algebra. Then, we consider the case of weak shuffle algebras. We prove that they can be equipped with a dendriform structure yet only two weak shuffle products can be considered as coming from a quadri-algebra.

\subsection{Dendriform algebras.}

4.1.1. Background.

Definition 4.1. A dendriform algebra is a vector space $\mathcal{D}$ equipped with two $\prec$ products $\succ$ such that $\forall x, y, z \in \mathcal{D}$,

$$
\begin{aligned}
& (x \prec y) \prec z=x \prec(y \prec z)+x \prec(y \succ z), \\
& (x \succ y) \prec z=x \succ(y \prec z), \\
& (x \prec y) \succ z+(x \succ y) \succ z=x \succ(y \succ z) .
\end{aligned}
$$

Theorem 4.2. Let $X$ be a countable alphabet and $\amalg$ be the classical shuffle product. We define $\prec$ and $\succ$ respectively by:

$$
a u \prec b v=a(u \amalg b v), a u \succ b v=b(a u \amalg v),
$$

for any letters $a$ and $b$ and any words $u$ and $v$. Then $(\mathbb{K}\langle X\rangle, \prec, \succ)$ is a dendriform algebra and for any words $u$ and $v$

$$
u \amalg v=u \prec v+u \succ v .
$$

Theorem 4.3. Let $X$ be a countable alphabet and $\amalg$ be the classical shuffle product. We define $\wedge$ and $\vee$ respectively by:

$$
u a \wedge v b=(u \amalg v b) a, u a \vee v b=(u a \amalg v) b,
$$


for any letters $a$ and $b$ and any words $u$ and $v$. Then $(\mathbb{K}\langle X\rangle, \wedge, \vee)$ is a dendriform algebra and for any words $u$ and $v$

$$
u \amalg v=u \wedge v+u \vee v
$$

\subsubsection{Weak shuffle products.}

Theorem 4.4. Let $X$ be a countable alphabet and $\square$ be a weak shuffle product such that $f_{1}(a \otimes a) \in\{0,1\}$ for any letter $a \in X$. We define the products $\prec$ and $\succ$ respectively by:

$$
a u \prec b v=f_{1}(a \otimes b) a(u \square b v), a u \succ b v=f_{2}(a \otimes b) b(a u \square v),
$$

for any letters $a$ and $b$ and any words $u$ and $v$. Then $(\mathbb{K}\langle X\rangle, \prec, \succ)$ is a dendriform algebra.

Proof. Let $\square$ be a weak shuffle product and let $a, b$ and $c$ be three letters of $X$. Then:

$$
\begin{aligned}
(a \prec b) \prec c & =f_{1}(a \otimes b) f_{1}(a \otimes c) f_{1}(b \otimes c) a b c+f_{1}(a \otimes b) f_{1}(a \otimes c) f_{2}(b \otimes c) a c b, \\
a \prec(b \square c) & =f_{1}(a \otimes b) f_{1}(b \otimes c) a b c+f_{1}(a \otimes c) f_{2}(b \otimes c) a c b, \\
(a \succ b) \prec c & =f_{2}(a \otimes b) f_{1}(b \otimes c) f_{1}(a \otimes c) b a c+f_{2}(a \otimes b) f_{1}(b \otimes c) f_{2}(a \otimes c) b c a, \\
a \succ(b \prec c) & =f_{2}(a \otimes b) f_{1}(b \otimes c) f_{1}(a \otimes c) b a c+f_{2}(a \otimes b) f_{1}(b \otimes c) f_{2}(a \otimes c) b c a, \\
(a \square b) \succ c & =f_{1}(a \otimes b) f_{2}(a \otimes c) c a b+f_{2}(a \otimes b) f_{2}(b \otimes c) c b a, \\
a \succ(b \succ c) & =f_{2}(b \otimes c) f_{2}(a \otimes c) f_{1}(a \otimes b) c a b+f_{2}(b \otimes c) f_{2}(a \otimes c) f_{2}(a \otimes b) c b a .
\end{aligned}
$$

Then $(a \succ b) \prec c=a \succ(b \prec c)$. If the three letters are all distinct or only two of them are equal or $a=b=c$ with $f_{1}(a \otimes a)=f_{2}(a \otimes a) \in\{0,1\}$ the relations given by Theorem 3.5 imply $(a \prec b) \prec c=a \prec(b \square c)$ and $(a \square b) \succ c=a \succ(b \succ c)$. If $a=b=c$ with $f_{1}(a \otimes a)=1-f_{2}(a \otimes a)$ then $(a \prec a) \prec a=a \prec(a \square a)$ and $(a \square a) \succ$ $a=a \succ(a \succ a)$ if and only if $f_{1}(a \otimes a) \in\{0,1\}$ and then $f_{1}(a \otimes a) f_{2}(a \otimes a)=0$.

We assume now there exists an integer $n \leq 3$ such that, for any non-empty words $u, v$ and $w$ with length $(u)+\operatorname{length}(v)+\operatorname{length}(w)=n$, relations $(u \prec v) \prec w=$ $u \prec(v \square w),(u \succ v) \prec w=u \succ(v \prec w)$ and $(u \square v) \succ w=u \succ(v \succ w)$ are satisfied.

Let $u, v$ and $w$ be three non-empty words such that length $(u)+\operatorname{length}(v)+$ length $(w)=n+1$. There exist three letters $a, b$ and $c$, not necessarily distinct and three words $u_{1}, v_{1}$ and $w_{1}$, not necessarily non-empty, such that $u=a u_{1}, v=b v_{1}$ and $w=c w_{1}$. Then 
(1)

$$
\begin{aligned}
(u \prec v) \prec w= & f_{1}(a \otimes b) f_{1}(a \otimes c) a\left[\left(u_{1} \square b v_{1}\right) \square c w_{1}\right]=f_{1}(a \otimes b) f_{1}(a \otimes c) a\left[u_{1} \square\left(b v_{1} \square c w_{1}\right)\right] \\
= & f_{1}(a \otimes b) f_{1}(a \otimes c) f_{1}(b \otimes c) a\left[u_{1} \square b\left(v_{1} \square c w_{1}\right)\right] \\
& +f_{1}(a \otimes b) f_{1}(a \otimes c) f_{2}(b \otimes c) a\left[u_{1} \square c\left(b v_{1} \square w_{1}\right)\right], \\
u \prec(v \square w)= & f_{1}(b \otimes c) f_{1}(a \otimes b) a\left[u_{1} \square b\left(v_{1} \square c w_{1}\right)\right] \\
& +f_{2}(b \otimes c) f_{1}(a \otimes c) f_{1}(a \otimes c) a\left[u_{1} \square c\left(b v_{1} \square w_{1}\right)\right] .
\end{aligned}
$$

$$
\begin{aligned}
& (u \succ v) \prec w=f_{2}(a \otimes b) f_{1}(b \otimes c) b\left[\left(a u_{1} \square v_{1}\right) \square c w_{1}\right], \\
& u \succ(v \prec w)=f_{1}(b \otimes c) f_{2}(a \otimes b) b\left[a u_{1} \square\left(v_{1} \square c w_{1}\right)\right] .
\end{aligned}
$$

$$
\begin{aligned}
(u \square v) \succ w= & f_{1}(a \otimes b) f_{2}(a \otimes c) c\left[a\left(u_{1} \square b v_{1}\right) \square c w_{1}\right]+f_{2}(a \otimes b) f_{2}(b \otimes c) c\left[b\left(a u_{1} \square v_{1}\right) \square c w_{1}\right], \\
u \succ(v \succ w)= & f_{2}(b \otimes c) f_{2}(a \otimes c) c\left[a u_{1} \square\left(b v_{1} \square w_{1}\right)\right]=f_{2}(b \otimes c) f_{2}(a \otimes c) c\left[\left(a u_{1} \square b v_{1}\right) \square w_{1}\right] \\
= & f_{2}(b \otimes c) f_{2}(a \otimes c) f_{1}(a \otimes b) c\left[a\left(u_{1} \square b v_{1}\right) \square w_{1}\right] \\
& +f_{2}(b \otimes c) f_{2}(a \otimes c) f_{2}(a \otimes b) c\left[b\left(a u_{1} \square v_{1}\right) \square w_{1}\right] .
\end{aligned}
$$

Thus, $(u \prec v) \prec w=u \prec(v \square w),(u \succ v) \prec w=u \succ(v \prec w)$ and $(u \square v) \succ w=$ $u \succ(v \succ w)$.

By considering the right hand side rather than the left hand side, we get the following definition and theorem.

Definition 4.5. Let $X$ be a countable alphabet. An end weak shuffle product on $\mathbb{K}\langle X\rangle$ is an associative and commutative product $\square_{E}$ such that for any $(a, b) \in(X)^{2}$ and any $(u, v) \in\left(X^{*}\right)^{2}$ then

$$
u a \square_{E} v b=f_{1, E}(a \otimes b)\left(u \square_{E} v b\right) a+f_{2, E}(a \otimes b)\left(u a \square_{E} v\right) b,
$$

where $f_{1, E}$ and $f_{2, E}$ are linear maps from $\mathbb{K} . X \otimes \mathbb{K} . X$ to $\mathbb{K}, u \square_{E} 0=0 \square_{E} u=0$ and $u \square_{E} 1=1 \square_{E} u=u$.

Theorem 4.6. Let $X$ be a countable alphabet and let $\square_{E}$ be an end weak shuffle product such that $f_{1, E}(a \otimes a) \in\{0,1\}$ for any letter $a \in X$. We define the products $\wedge$ and $\vee$ by:

$$
u a \wedge v b=f_{1, E}(a \otimes b)\left(u \square_{E} v b\right) a, a u \vee b v=f_{2, E}(a \otimes b)\left(u a \square_{E} v\right) b,
$$

for any letters $a$ and $b$ and any words $u$ and $v$. Then $(\mathbb{K}\langle X\rangle, \wedge, \vee)$ is a dendriform algebra. 
Remark 4.7. Let $\alpha$ be a real number. Let $\square$ be the weak shuffle product satisfying $f_{1}(a \otimes a)=1-f_{2}(a \otimes a)=\alpha$ for a unique letter $a$. Even if $\square$ does not depend on the value of $\alpha$, to express the algebra as a dendriform algebra the assumption $\alpha \in\{0,1\}$ is necessary.

\subsection{Quadri-algebras.}

\subsubsection{Background.}

Definition 4.8. A quadri-algebra is $\mathcal{Q}$ is a vector space equipped with four products $\searrow, \nearrow, \nwarrow$ and $\swarrow$ such that: for any $x, y, z \in \mathcal{Q}$,

$$
\begin{array}{rlrl}
(x \nwarrow y) \nwarrow z & =x \nwarrow(y \cdot z), & & (x \nearrow y) \nwarrow z=x \nearrow(y \prec z), \\
(x \swarrow y) \nwarrow z & =x \swarrow(y \wedge z), & & (x \searrow y) \nwarrow z=x \searrow(y \nwarrow z), \\
(x \prec y) \swarrow z=x \swarrow(y \vee z), & & (x \succ y) \swarrow z=x \searrow(y \swarrow z),
\end{array}
$$

and

$$
\begin{aligned}
& (x \wedge y) \nearrow z=x \nearrow(y \succ z), \\
& (x \vee y) \nearrow z=x \searrow(y \nearrow z), \\
& (x \cdot y) \searrow z=x \searrow(y \searrow z)
\end{aligned}
$$

where

$$
\begin{aligned}
& x \prec y=x \nwarrow y+x \swarrow y, \quad x \wedge y=x \nearrow y+x \nwarrow y, \\
& x \succ y=x \nearrow y+x \searrow y, \quad x \vee y=x \searrow y+x \swarrow y,
\end{aligned}
$$

and

$$
x \cdot y=x \nwarrow y+x \swarrow y+x \nearrow y+x \searrow y=x \prec y+x \succ y=x \wedge y+x \vee y .
$$

Theorem 4.9. Let $X$ be a countable alphabet and let $\amalg$ be the classical shuffle product. The products $\searrow, \nearrow, \nwarrow$ and $\swarrow$ are defined as follow:

$$
\begin{aligned}
& a u c \nwarrow b v d=a(u \amalg b v d) c, a u c \swarrow b v d=a(u c \amalg b v) d, \\
& a u c \nearrow b v d=b(a u \amalg v d) c, a u c \searrow b v d=b(a u c \varpi v) d
\end{aligned}
$$

for any letters $a, b, c$ and $d$ and any words $u$ and $v$. Then $(\mathbb{K}\langle X\rangle, \searrow, \nearrow, \nwarrow, \swarrow)$ is a quadri-algebra. 
Proof. It is proved in [1, Section 1.8]. The main ingredient of the proof is the following statement: for any letters $a, b, c$ and $d$ and any words $u$ and $v$ we have

$$
a u c \amalg b v d=a(u c \amalg b v d)+b(a u c \amalg v d)=(a u \amalg b v d) c+(a u c \amalg b v) d .
$$

4.2.2. Weak shuffle algebras.

Proposition 4.10. Let $X$ be a countable alphabet of cardinality at least 2. Let $\square$ be a weak shuffle product. There exists an end weak shuffle product $\square_{E}$ such that $\square=\square_{E}$ if, and only if, $\square$ is the null product or the classical shuffle product.

Proof. It is sufficient to prove the proposition for an alphabet of cardinality 2 and assume images of functions $f_{1}, f_{2}, f_{1, E}$ and $f_{2, E}$ are subsets of $\{0,1\}$. Let $C$ be the 6-tuple $C=\left(f_{1}(a \otimes b), f_{1}(b \otimes a), f_{1}(a \otimes a), f_{2}(a \otimes a), f_{1}(b \otimes b), f_{2}(b \otimes b)\right)$.

Case $C=(1,0,0,0,0,0)$ : If $\square=\square_{E}$ then

$$
\begin{aligned}
a \square_{E} b a & =\left(f_{1, E}(a \otimes a)+f_{2, E}(a \otimes a) f_{1, E}(a \otimes b)\right) b a a+f_{2, E}(a \otimes a) f_{1, E}(b \otimes a) a b a \\
& =a \square b a=a b a .
\end{aligned}
$$

Thus $f_{2, E}(a \otimes a)=1$ and then $a \square_{E} a=\left(f_{1, E}(a \otimes a)+1\right) a a \neq 0$ and yet $a \square a=0$. Contradiction.

Cases $C=(1,0,1,1,0,0)$ and $C=(1,0,1,0,0,0)$ : We recall that these two cases are isomorphic. If $\square=\square_{E}$ then

$$
\begin{aligned}
a \square_{E} b a & =\left(f_{1, E}(a \otimes a)+f_{2, E}(a \otimes a) f_{1, E}(a \otimes b)\right) b a a+f_{2, E}(a \otimes a) f_{1, E}(b \otimes a) a b a \\
& =\left(f_{1, E}(a \otimes a) f_{1, E}(a \otimes b)+f_{2, E}(a \otimes a)\right) b a a+f_{1, E}(a \otimes a) f_{1, E}(b \otimes a) a b a \\
& =b a \square_{E} a=a \square b a=a b a .
\end{aligned}
$$

Thus $f_{1, E}(a \otimes a)=f_{2, E}(a \otimes a)=f_{1, E}(b \otimes a)=1$ and $f_{1, E}(a \otimes b)=-1$. Contradiction.

Cases $C=(1,0,1,0,1,1)$ and $C=(1,0,1,1,1,1)$ : The same calculations as in the previous case answer the question.

Case $C=(1,0,0,0,1,1)$ : If $\square=\square_{E}$ then

$$
\begin{aligned}
b a \square_{E} b & =f_{1, E}(a \otimes b)\left(f_{1, E}(b \otimes b)+f_{2, E}(b \otimes b)\right) b b a+f_{1, E}(b \otimes a) b a b \\
& =b a \square b=b b a+b a b .
\end{aligned}
$$

Thus $f_{1, E}(a \otimes b)=f_{1, E}(b \otimes a)=f_{1, E}(a \otimes a)=f_{2, E}(a \otimes a)=f_{1, E}(b \otimes b)=$ $f_{2, E}(b \otimes b)=1$ with $f_{1, E}(b \otimes b)+f_{2, E}(b \otimes b)=1$. Contradiction. 
Cases $C=(0,0,1,1,0,0)$ : If $\square=\square_{E}$ then

$$
\begin{aligned}
a b \square_{E} a & =f_{1, E}(b \otimes a)\left(f_{1, E}(a \otimes a)+f_{2, E}(a \otimes a)\right) a a b+f_{1, E}(a \otimes b) a b a \\
& =a b \square a=a a b .
\end{aligned}
$$

Thus $f_{1, E}(a \otimes b)=0, f_{1, E}(b \otimes a)=1$ and $f_{1, E}(a \otimes a)+f_{2, E}(a \otimes a)=1$. Contradiction.

Cases $C=(0,0,1,1,1,1)$ : The same calculations as in the previous case answer the question.

Corollary 4.11. The construction used in Theorem 4.9 does not lead to a quadrialgebra structure on a weak shuffle product $\square$ except if $\square$ is the null shuffle or the classical shuffle.

\section{Relations on weak stuffle products}

Proposition 5.1. Let $X$ be a countable alphabet, let $a, b$ and $c$ be three distinct letters in $X$ and $\square$ a weak stuffle product. Then:

(1) By using the maps $f_{1}$ and $f_{2}$ coming from $\square$, we define the product $\square^{\prime}$ by: $a u \square^{\prime} b v=f_{1}(a \otimes b) a\left(u \square^{\prime} b v\right)+f_{2}(a \otimes b) b\left(a u \square^{\prime} v\right)$ for any letters $a$ and $b$ and any words $u$ and $v$. The product $\square^{\prime}$ is a weak shuffle product.

(2) The function $f_{3}$ is associative and commutative.

(3) If $f_{3}(a \otimes a) \neq 0$ then $f_{1}(a \otimes a)=f_{2}(a \otimes a) \in\{0,1\}$.

(4) If $f_{3}(a \otimes b) \neq 0$ then $f_{1}(a \otimes a)=f_{2}(a \otimes a) \in\{0,1\}$ and $f_{1}(b \otimes b)=f_{2}(b \otimes b) \in$ $\{0,1\}$.

(5) If $f_{3}(a \otimes a) \in \mathbb{K}^{*} a$ then $f_{1}(b \otimes a) \in\{0,1\}$.

(6) If $f_{3}(a \otimes a) \in \mathbb{K}^{*} b$ then

(a) If $f_{3}(a \otimes b) \neq 0$ or $f_{3}(b \otimes b) \neq 0$ or there exists $x \in X \backslash\{a, b\}$ such that $f_{3}(b \otimes x) \neq 0$ then $f_{1}(a \otimes a)=f_{2}(a \otimes a)=f_{1}(b \otimes b)=f_{2}(b \otimes b)=$ $f_{1}(a \otimes b)=f_{1}(b \otimes a) \in\{0,1\}$.

(b) If $f_{3}(a \otimes b)=0$ and $f_{3}(b \otimes b)=0$ then

(i) either $f_{1}(a \otimes a)=f_{2}(a \otimes a)=f_{1}(b \otimes b)=f_{2}(b \otimes b)=f_{1}(a \otimes b)=$ $f_{1}(b \otimes a) \in\{0,1\}$

(ii) or $f_{1}(a \otimes a)=f_{2}(a \otimes a)=f_{1}(b \otimes a)=1, f_{1}(b \otimes b)+f_{2}(b \otimes b)=1$ and $f_{1}(a \otimes b)=0$.

(c) For any $x \in X \backslash\{a, b\}$ then

(i) $f_{1}(a \otimes x)=f_{1}(b \otimes x)$,

(ii) $f_{1}^{2}(x \otimes a)=f_{1}(x \otimes b)$.

(7) If $f_{3}(a \otimes b) \in \mathbb{K}^{*} a$ then:

(a) $f_{1}(b \otimes a)=f_{1}(a \otimes a) f_{1}(a \otimes b)=f_{1}(b \otimes a) f_{1}(b \otimes b)$. 
(b) $f_{1}(a \otimes b)=f_{1}(b \otimes b)$.

(c) For any $x \in X \backslash\{a, b\}$ such that $f_{3}(b \otimes x) \notin \mathbb{K}^{*} x$ then

(i) $f_{1}(a \otimes x)=f_{1}(b \otimes x)$,

(ii) $f_{1}(x \otimes a)\left[1-f_{1}(x \otimes b)\right]=0$.

(d) For any $x \in X \backslash\{a, b\}$ such that $f_{3}(b \otimes x) \in \mathbb{K}^{*} x$ then

(i) $f_{1}(b \otimes a)=f_{1}(x \otimes a) f_{1}(x \otimes b)$,

(ii) $f_{1}(b \otimes x)=f_{1}(a \otimes b) f_{1}(a \otimes x)$,

(8) If $f_{3}(a \otimes b) \in \mathbb{K}^{*} c$ then:

(a) $f_{1}(c \otimes c)=f_{2}(c \otimes c) \in\{0,1\}$.

(b) $f_{1}(b \otimes a)=f_{1}(c \otimes a)=f_{1}(a \otimes a)$.

(c) $f_{1}(a \otimes b)=f_{1}(c \otimes b)=f_{1}(b \otimes b)$.

(d) $f_{1}(a \otimes c)=f_{1}(a \otimes a) f_{1}(b \otimes b)=f_{1}(b \otimes c)=f_{1}(c \otimes c)$.

Proof. (1) Let $a$ and $b$ be two letters and let $u$ and $v$ be two words. By using words of length length $(u)+$ length $(v)+2$ appearing in $a u \square b v$, we get the statement. In the sequel, the use of the relations given in Theorem 3.5 is implied.

(2) By using words of length 1 appearing in $x \square y, x \square y,(x \square y) \square z$ and $x \square(y \square z)$ for any letters $x, y, z$, we prove that the function $f_{3}$ is associative and commutative.

(3) We assume $f_{3}(a \otimes a) \neq 0$. Since $a \square a a=a a \square a$ and $(a \square a) \square a a=a \square(a \square a a)$ then $f_{1}(a \otimes a)=f_{2}(a \otimes a) \in\{0,1\}$.

(4) We assume $f_{3}(a \otimes b) \neq 0$. Since $a \square a b=a b \square a, b \square b a=b a \square b,(a \square b) \square a=$ $(a \square a) \square b$ and $(b \square a) \square b=(b \square b) \square a$ then $f_{1}(a \otimes a)=f_{2}(a \otimes a) \in\{0,1\}$ and $f_{1}(b \otimes b)=f_{2}(b \otimes b) \in\{0,1\}$.

(5) This item is proved by using $(a \square a) \square b=(a \square b) \square a$ and $a \square(a \square b a)=(a \square a) \square b a$.

(6) We assume $f_{3}(a \otimes a) \in \mathbb{K}^{*} b$.

(a) If $f_{3}(a \otimes b) \neq 0$ or $f_{3}(b \otimes b) \neq 0$, since $f_{1}(a \otimes a)=f_{2}(a \otimes a) \in\{0,1\}$, $f_{1}(b \otimes b)=f_{2}(b \otimes b) \in\{0,1\},(a \square b) \square a=(a \square a) \square b$ and $(a \square a) \square a a=$ $a \square(a \square a a)$, then $f_{1}(a \otimes a)=f_{2}(a \otimes a)=f_{1}(b \otimes b)=f_{2}(b \otimes b)=$ $f_{1}(a \otimes b)=f_{1}(b \otimes a) \in\{0,1\}$.

(b) If $f_{3}(a \otimes b)=0$ and $f_{3}(b \otimes b)=0$, since $f_{1}(a \otimes a)=f_{2}(a \otimes a) \in\{0,1\}$, $(a \square b) \square a=(a \square a) \square b$ and $(a \square a) \square a a=a \square(a \square a a)$ then we prove the relations.

(c) This item is proved thanks to the relation $(a \square b) \square a=(a \square a) \square b$.

(7) We assume $f_{3}(a \otimes b) \in \mathbb{K}^{*} a$.

(a) This item is proved by using $f_{1}(a \otimes a)=f_{2}(a \otimes a) \in\{0,1\}, f_{1}(b \otimes b)=$ $f_{2}(b \otimes b) \in\{0,1\},(a \square b) \square a=(a \square a) \square b$ and $(b \square a) \square b=(b \square b) \square a$. 
(b) By using $(b \square b) \square a=(b \square a) \square b$ and $(a \square b) \square b a=a \square(b \square b a)$ we prove $f_{1}(a \otimes b)=f_{1}(b \otimes b)$.

(c) Those two subitems are proven by using $(a \square b) \square x=(a \square x) \square b=$ $(b \square x) \square a$.

(d) Those two subitems are proven by using $(a \square b) \square x=(a \square x) \square b=$ $(b \square x) \square a$.

(8) We assume $f_{3}(a \otimes b) \in \mathbb{K}^{*} c$. Then $f_{1}(a \otimes a)=f_{2}(a \otimes a) \in\{0,1\}$ and $f_{1}(b \otimes b)=f_{2}(b \otimes b) \in\{0,1\}$. By using the relations $(a \square b) \square c=(a \square c) \square b=$ $(b \square c) \square a,(a \square b) \square b=(b \square b) \square a,(b \square a) \square a=(a \square a) \square b,(a \square b) \square a a=a \square(b \square a a)=$ $b \square(a \square a a)$ and $(b \square a) \square b b=b \square(a \square b b)=a \square(b \square b b)$ we prove all subitems.

Examples 5.2. (1) The $q$-shuffle product associated to the Schlesinger-Zudilin model is the weak stuffle product where $f_{1}(y \otimes p)=f_{1}(y \otimes y)=f_{1}(p \otimes p)=$ $f_{2}(p \otimes p)=1, f_{1}(p \otimes y)=f_{2}(y \otimes y)=0, f_{3}(p \otimes p)=p, f_{3}(y \otimes p)=$ $f_{3}(y \otimes y)=0$.

(2) The $q$-shuffle product associated to the Bradley-Zhao model is the weak stuffle product where $f_{1}(y \otimes p)=f_{1}(y \otimes \bar{p})=f_{1}(p \otimes \bar{p})=f_{1}(\bar{p} \otimes p)=$ $f_{1}(p \otimes p)=f_{2}(p \otimes p)=f_{1}(\bar{p} \otimes \bar{p})=f_{2}(\bar{p} \otimes \bar{p})=f_{1}(y \otimes y)=1, f_{1}(p \otimes y)=$ $f_{1}(\bar{p} \otimes y)=f_{2}(y \otimes y)=0, f_{3}(p \otimes p)=p, f_{3}(\bar{p} \otimes \bar{p})=-\bar{p} f_{3}(y \otimes p)=$ $f_{3}(y \otimes y)=f_{3}(y \otimes \bar{p})=f_{3}(p \otimes \bar{p})=0$.

Corollary 5.3. Let $X=\left\{x_{1}, \ldots, x_{n} \ldots\right\}$ be an infinite countable alphabet. We assume $\square$ is a weak stuffle product such that $f_{3}\left(x_{i} \otimes x_{j}\right) \in \mathbb{K}^{*} x_{i+j}$ for any positive integers $i$ and $j$. Then, the underlying weak shuffle produit is either the null shuffle product or the classical stuffle product i.e. $\left(f_{1} \equiv 0\right.$ and $\left.f_{2} \equiv 0\right)$ or $\left(f_{1}(a \otimes b)=1\right.$ and $f_{2}(a \otimes b)=1$ for any letters $a$ and $\left.b\right)$.

Proof. We use an inductive proof. First of all, since $f_{3}\left(x_{i} \otimes x_{i}\right) \neq 0$ for any positive integer $i$, we have $f_{1}\left(x_{i} \otimes x_{i}\right)=f_{2}\left(x_{i} \otimes x_{i}\right)$. Besides, $f_{3}\left(x_{1} \otimes x_{1}\right)=x_{2} \neq x_{1}$ and $f_{3}\left(x_{2} \otimes x_{2}\right) \neq 0$, so $f_{1}\left(x_{1} \otimes x_{1}\right)=f_{2}\left(x_{1} \otimes x_{1}\right)=f_{1}\left(x_{2} \otimes x_{2}\right)=f_{2}\left(x_{2} \otimes x_{2}\right)=$ $f_{1}\left(x_{1} \otimes x_{2}\right)=f_{1}\left(x_{2} \otimes x_{1}\right) \in\{0,1\}$.

We assume there exists $n \in \mathbb{N}^{*}$ such that $n \geq 2$ and $f_{1}\left(x_{1} \otimes x_{1}\right)=f_{1}\left(x_{1} \otimes x_{m}\right)$ for any $m \in \llbracket 1, n \rrbracket$. Then, $f_{3}\left(x_{1} \otimes x_{n}\right)=x_{n+1}$ and $f_{1}\left(x_{1} \otimes x_{n+1}\right)=f_{1}\left(x_{1} \otimes x_{1}\right) f_{1}\left(x_{1} \otimes\right.$ $\left.x_{n}\right)=f_{1}\left(x_{1} \otimes x_{1}\right)$. Thus, $f_{1}\left(x_{1} \otimes x_{1}\right)=f_{1}\left(x_{1} \otimes x_{n}\right)$ for any positive integer $n$.

We assume now there exists $k \in \mathbb{N}^{*}$ such that $f_{1}\left(x_{1} \otimes x_{1}\right)=f_{1}\left(x_{i} \otimes x_{j}\right)$ for any $i \in \llbracket 1, k \rrbracket$ and any positive integer $j$. For any $i \in \llbracket 1, k \rrbracket$, we know $f_{3}\left(x_{i} \otimes x_{k+1-i}\right)=$ $x_{k+1}$ so, $f_{1}\left(x_{k+1} \otimes x_{i}\right)=f_{1}\left(x_{k+1-i} \otimes x_{i}\right)=f_{1}\left(x_{1} \otimes x_{1}\right)$. Besides, we know

$$
f_{1}\left(x_{k+1} \otimes x_{k+1}\right)=f_{2}\left(x_{k+1} \otimes x_{k+1}\right)=f_{1}\left(x_{1} \otimes x_{k+1}\right)=f_{1}\left(x_{1} \otimes x_{1}\right) .
$$


Since $f_{3}\left(x_{k+1} \otimes x_{1}\right)=x_{k+2}$, we have $f_{1}\left(x_{k+1} \otimes x_{k+2}\right)=f_{1}\left(x_{1} \otimes x_{k+2}\right)=f_{1}\left(x_{1} \otimes x_{1}\right)$. We assume there exists a positive integer $j$ such that $f_{1}\left(x_{k+1} \otimes x_{k+1+p}\right)=f_{1}\left(x_{1} \otimes x_{1}\right)$ for any $p \in \llbracket 1, j \rrbracket$. As $f_{3}\left(x_{k+1} \otimes x_{j+1}\right)=x_{k+j+2}$ then

$$
f_{1}\left(x_{k+1} \otimes x_{k+j+2}\right)=f_{1}\left(x_{k+1} \otimes x_{k+1}\right) f_{1}\left(x_{k+1} \otimes x_{j+1}\right)=f_{1}\left(x_{1} \otimes x_{1}\right) .
$$

Finally, $\left(f_{1} \equiv 0\right.$ and $\left.f_{2} \equiv 0\right)$ or $\left(f_{1}(a \otimes b)=1\right.$ and $f_{2}(a \otimes b)=1$ for any letters $a$ and $b)$.

By using the commutativity and the associativity of $k_{3}$ we have:

Lemma 5.4. Let $X=\{a, b\}$ be an alphabet of cardinality 2 and let $\square$ be a weak stuffle product. The map $f_{3}$ is one of the following:

(1) There exists $(\lambda, \mu) \in\left(\mathbb{K}^{*}\right)^{2}$ such that $f_{3}(a \otimes a)=\lambda b, f_{3}(a \otimes b)=\mu a$ and $f_{3}(b \otimes b)=\mu b$.

(2) There exists $(\lambda, \mu) \in\left(\mathbb{K}^{*}\right)^{2}$ such that $f_{3}(a \otimes a)=\lambda a, f_{3}(a \otimes b)=\mu a$ and $f_{3}(b \otimes b)=\frac{\mu^{2}}{\lambda} a$.

(3) There exists $(\lambda, \mu) \in\left(\mathbb{K}^{*}\right)^{2}$ such that $f_{3}(a \otimes a)=\lambda a, f_{3}(a \otimes b)=\mu a$ and $f_{3}(b \otimes b)=\mu b$.

(4) There exists $(\lambda, \mu) \in\left(\mathbb{K}^{*}\right)^{2}$ such that $f_{3}(a \otimes a)=0, f_{3}(a \otimes b)=\mu a$ and $f_{3}(b \otimes b)=\lambda b$.

(5) There exists $(\lambda, \mu) \in\left(\mathbb{K}^{*}\right)^{2}$ such that $f_{3}(a \otimes a)=\lambda a, f_{3}(a \otimes b)=0$ and $f_{3}(b \otimes b)=\mu b$.

(6) There exists $\lambda \in \mathbb{K}^{*}$ such that $f_{3}(a \otimes a)=\lambda b, f_{3}(a \otimes b)=0$ and $f_{3}(b \otimes b)=0$.

(7) There exists $\lambda \in \mathbb{K}^{*}$ such that $f_{3}(a \otimes a)=\lambda a, f_{3}(a \otimes b)=0$ and $f_{3}(b \otimes b)=0$.

(8) The map $f_{3}$ is the null map.

By using Proposition 5.1 we have:

Proposition 5.5. Let $X=\{a, b\}$ be an alphabet of cardinality 2 and let $\square$ be a weak stuffle product. In the previous lemma, if $f_{3}$ satisfies

(1) Item (1) or item (2), then there are two cases:

- $f_{1}(a \otimes b)=1$ and $f_{2}(a \otimes b)=1$ for any $(a, b) \in X^{2}$,

- $f_{1} \equiv 0$ and $f_{2} \equiv 0$.

(2) Item (3) or item (4), then there are four cases:

- $f_{1}(a \otimes b)=1$ and $f_{2}(a \otimes b)=1$ for any $(a, b) \in X^{2}$,

- $f_{1} \equiv 0$ and $f_{2} \equiv 0$,

- $f_{1}(b \otimes a)=f_{1}(a \otimes b)=f_{1}(b \otimes b)=f_{2}(b \otimes b)=0$ and $f_{1}(a \otimes a)=$ $f_{2}(a \otimes a)=1$,

- $f_{1}(a \otimes b)=f_{1}(b \otimes b)=f_{2}(b \otimes b)=1$ and $f_{1}(b \otimes a)=f_{1}(a \otimes a)=$ $f_{2}(a \otimes a)=0$. 
(3) Item (5), then we have:

- $f_{1}(a \otimes b) \in\{0,1\}$,

- $f_{1}(b \otimes a) \in\{0,1\}$,

- $f_{1}(a \otimes a)=f_{2}(a \otimes a) \in\{0,1\}$,

- $f_{1}(b \otimes b)=f_{2}(b \otimes b) \in\{0,1\}$.

(4) Item (6), then there are three cases:

- $f_{1}(a \otimes b)=1$ and $f_{2}(a \otimes b)=1$ for any $(a, b) \in X^{2}$,

- $f_{1} \equiv 0$ and $f_{2} \equiv 0$,

- $f_{1}(a \otimes a)=f_{2}(a \otimes a)=f_{1}(b \otimes a)=1, f_{1}(a \otimes b)=0$ and $f_{1}(b \otimes b)+$ $f_{2}(b \otimes b)=1$

(5) Item (7), then we have:

- $f_{1}(b \otimes a) \in\{0,1\}$,

- $f_{1}(a \otimes a)=f_{2}(a \otimes a) \in\{0,1\}$.

(6) Item (8), then we give the answer in Theorem 3.5.

Lemma 5.6. Let $X=\{a, b, c\}$ be an alphabet of cardinality 3 and let $\square$ be a weak stuffle product. The map $f_{3}$ is one of the following:

(1) There exists $(\lambda, \gamma, \mu) \in\left(\mathbb{K}^{*}\right)^{3}$ such that $\gamma \mu=\lambda^{2}, f_{3}(a \otimes b)=\lambda c, f_{3}(a \otimes c)=$ $\lambda a, f_{3}(b \otimes c)=\lambda b, f_{3}(a \otimes a)=\gamma b, f_{3}(b \otimes b)=\mu a$ and $f_{3}(c \otimes c)=\lambda c$.

(2) There exists $(\lambda, \gamma, \mu) \in\left(\mathbb{K}^{*}\right)^{3}$ such that $f_{3}(a \otimes b)=\lambda c, f_{3}(a \otimes c)=\gamma c$, $f_{3}(b \otimes c)=\mu c, f_{3}(a \otimes a)=\gamma a, f_{3}(b \otimes b)=\frac{\lambda \mu}{\gamma} a$ and $f_{3}(c \otimes c)=\frac{\gamma \mu}{\lambda} c$.

(3) There exists $(\lambda, \gamma, \mu) \in\left(\mathbb{K}^{*}\right)^{3}$ such that $f_{3}(a \otimes b)=\lambda c, f_{3}(a \otimes c)=\gamma c$, $f_{3}(b \otimes c)=\mu c, f_{3}(a \otimes a)=\gamma a, f_{3}(b \otimes b)=\mu b$ and $f_{3}(c \otimes c)=\frac{\gamma \mu}{\lambda} c$.

(4) There exists $(\lambda, \gamma, \mu) \in\left(\mathbb{K}^{*}\right)^{3}$ such that $f_{3}(a \otimes b)=\lambda c, f_{3}(a \otimes c)=\gamma c$, $f_{3}(b \otimes c)=\mu c, f_{3}(a \otimes a)=\gamma a, f_{3}(b \otimes b)=\frac{\lambda \mu}{\gamma} c$ and $f_{3}(c \otimes c)=\frac{\gamma \mu}{\lambda} c$.

(5) There exists $(\lambda, \gamma, \mu) \in\left(\mathbb{K}^{*}\right)^{3}$ such that $f_{3}(a \otimes b)=\lambda c, f_{3}(a \otimes c)=\gamma c$, $f_{3}(b \otimes c)=\mu c, f_{3}(a \otimes a)=\frac{\gamma^{2}}{\mu} b, f_{3}(b \otimes b)=\frac{\lambda \mu}{\gamma} c$ and $f_{3}(c \otimes c)=\frac{\gamma \mu}{\lambda} c$.

(6) There exists $(\lambda, \gamma, \mu) \in\left(\mathbb{K}^{*}\right)^{3}$ such that $f_{3}(a \otimes b)=\lambda c, f_{3}(a \otimes c)=\gamma c$, $f_{3}(b \otimes c)=\mu c, f_{3}(a \otimes a)=\frac{\lambda \gamma}{\mu} c, f_{3}(b \otimes b)=\frac{\mu^{2}}{\gamma} a$ and $f_{3}(c \otimes c)=\frac{\gamma \mu}{\lambda} c$.

(7) There exists $(\lambda, \gamma, \mu) \in\left(\mathbb{K}^{*}\right)^{3}$ such that $f_{3}(a \otimes b)=\lambda c, f_{3}(a \otimes c)=\gamma c$, $f_{3}(b \otimes c)=\mu c, f_{3}(a \otimes a)=\frac{\lambda \gamma}{\mu} c, f_{3}(b \otimes b)=\mu b$ and $f_{3}(c \otimes c)=\frac{\gamma \mu}{\lambda} c$.

(8) There exists $(\lambda, \gamma, \mu) \in\left(\mathbb{K}^{*}\right)^{3}$ such that $f_{3}(a \otimes b)=\lambda c, f_{3}(a \otimes c)=\gamma c$, $f_{3}(b \otimes c)=\mu c, f_{3}(a \otimes a)=\frac{\lambda \gamma}{\mu} c, f_{3}(b \otimes b)=\frac{\lambda \mu}{\gamma} c$ and $f_{3}(c \otimes c)=\frac{\gamma \mu}{\lambda} c$.

(9) There exists $(\lambda, \gamma) \in\left(\mathbb{K}^{*}\right)^{2}$ such that $f_{3}(a \otimes b)=\lambda c, f_{3}(a \otimes c)=\gamma c$, $f_{3}(b \otimes c)=0, f_{3}(a \otimes a)=\gamma a, f_{3}(b \otimes b)=0$ and $f_{3}(c \otimes c)=0$.

(10) There exists $\lambda \in \mathbb{K}^{*}$ such that $f_{3}(a \otimes b)=\lambda b, f_{3}(a \otimes c)=\lambda c, f_{3}(b \otimes c)=0$, $f_{3}(a \otimes a)=\lambda a, f_{3}(b \otimes b)=0$ and $f_{3}(c \otimes c)=0$. 
(11) There exists $(\lambda, \gamma) \in\left(\mathbb{K}^{*}\right)^{2}$ such that $f_{3}(a \otimes b)=\lambda b, f_{3}(a \otimes c)=\lambda c$, $f_{3}(b \otimes c)=0, f_{3}(a \otimes a)=\lambda a, f_{3}(b \otimes b)=0$ and $f_{3}(c \otimes c)=\gamma b$.

(12) There exists $(\lambda, \gamma) \in\left(\mathbb{K}^{*}\right)^{2}$ such that $f_{3}(a \otimes b)=\lambda b, f_{3}(a \otimes c)=\lambda c$, $f_{3}(b \otimes c)=0, f_{3}(a \otimes a)=\lambda a, f_{3}(b \otimes b)=0$ and $f_{3}(c \otimes c)=\gamma c$.

(13) There exists $(\lambda, \gamma, \mu) \in\left(\mathbb{K}^{*}\right)^{3}$ such that $f_{3}(a \otimes b)=\lambda b, f_{3}(a \otimes c)=\lambda c$, $f_{3}(b \otimes c)=0, f_{3}(a \otimes a)=\lambda a, f_{3}(b \otimes b)=\gamma b$ and $f_{3}(c \otimes c)=\mu c$.

(14) There exists $(\lambda, \gamma, \mu) \in\left(\mathbb{K}^{*}\right)^{3}$ such that $f_{3}(a \otimes b)=\lambda c, f_{3}(a \otimes c)=0$, $f_{3}(b \otimes c)=0, f_{3}(a \otimes a)=\gamma c, f_{3}(b \otimes b)=\mu c$ and $f_{3}(c \otimes c)=0$.

(15) There exists $(\lambda, \gamma) \in\left(\mathbb{K}^{*}\right)^{2}$ such that $f_{3}(a \otimes b)=\lambda c, f_{3}(a \otimes c)=0, f_{3}(b \otimes c)=$ $0, f_{3}(a \otimes a)=\gamma b, f_{3}(b \otimes b)=0$ and $f_{3}(c \otimes c)=0$.

(16) There exists $\lambda \in \mathbb{K}^{*}$ such that $f_{3}(a \otimes b)=\lambda c, f_{3}(a \otimes c)=0, f_{3}(b \otimes c)=0$, $f_{3}(a \otimes a)=0, f_{3}(b \otimes b)=0$ and $f_{3}(c \otimes c)=0$.

(17) There exists $(\lambda, \gamma, \mu, \tau) \in\left(\mathbb{K}^{*}\right)^{4}$ such that $\gamma \mu=\lambda^{2}, f_{3}(a \otimes b)=\lambda a, f_{3}(a \otimes$ $c)=0, f_{3}(b \otimes c)=0, f_{3}(a \otimes a)=\gamma a, f_{3}(b \otimes b)=\mu a$ and $f_{3}(c \otimes c)=\tau c$.

(18) There exists $(\lambda, \gamma, \tau) \in\left(\mathbb{K}^{*}\right)^{3}$ such that $f_{3}(a \otimes b)=\lambda a, f_{3}(a \otimes c)=0$, $f_{3}(b \otimes c)=0, f_{3}(a \otimes a)=\gamma a, f_{3}(b \otimes b)=\lambda b$ and $f_{3}(c \otimes c)=\tau c$.

(19) There exists $(\lambda, \gamma, \tau) \in\left(\mathbb{K}^{*}\right)^{3}$ such that $f_{3}(a \otimes b)=\lambda a, f_{3}(a \otimes c)=0$, $f_{3}(b \otimes c)=0, f_{3}(a \otimes a)=\gamma b, f_{3}(b \otimes b)=\lambda b$ and $f_{3}(c \otimes c)=\tau c$.

(20) There exists $(\lambda, \gamma) \in\left(\mathbb{K}^{*}\right)^{2}$ such that $f_{3}(a \otimes b)=\lambda a, f_{3}(a \otimes c)=0, f_{3}(b \otimes$ $c)=0, f_{3}(a \otimes a)=\gamma c, f_{3}(b \otimes b)=\lambda b$ and $f_{3}(c \otimes c)=0$.

(21) There exists $(\lambda, \tau) \in\left(\mathbb{K}^{*}\right)^{2}$ such that $f_{3}(a \otimes b)=\lambda a, f_{3}(a \otimes c)=0, f_{3}(b \otimes$ $c)=0, f_{3}(a \otimes a)=0, f_{3}(b \otimes b)=\lambda b$ and $f_{3}(c \otimes c)=\tau c$.

(22) There exists $\left(\lambda, \gamma, \mu \in\left(\mathbb{K}^{*}\right)^{3}\right.$ such that $\gamma \mu=\lambda^{2}, f_{3}(a \otimes b)=\lambda a, f_{3}(a \otimes c)=$ $0, f_{3}(b \otimes c)=0, f_{3}(a \otimes a)=\gamma a, f_{3}(b \otimes b)=\mu a$ and $f_{3}(c \otimes c)=0$.

(23) There exists $(\lambda, \gamma, \tau) \in\left(\mathbb{K}^{*}\right)^{3}$ such that $f_{3}(a \otimes b)=\lambda a, f_{3}(a \otimes c)=0$, $f_{3}(b \otimes c)=0, f_{3}(a \otimes a)=\gamma a, f_{3}(b \otimes b)=\lambda b$ and $f_{3}(c \otimes c)=0$.

(24) There exists $(\lambda, \gamma) \in\left(\mathbb{K}^{*}\right)^{2}$ such that $f_{3}(a \otimes b)=\lambda a, f_{3}(a \otimes c)=0, f_{3}(b \otimes$ $c)=0, f_{3}(a \otimes a)=\gamma b, f_{3}(b \otimes b)=\lambda b$ and $f_{3}(c \otimes c)=0$.

(25) There exists $\lambda \in \mathbb{K}^{*}$ such that $f_{3}(a \otimes b)=\lambda a, f_{3}(a \otimes c)=0, f_{3}(b \otimes c)=0$, $f_{3}(a \otimes a)=0, f_{3}(b \otimes b)=\lambda b$ and $f_{3}(c \otimes c)=0$.

(26) There exists $(\lambda, \gamma, \mu) \in\left(\mathbb{K}^{*}\right)^{3}$ such that $f_{3}(a \otimes b)=0, f_{3}(a \otimes c)=0$, $f_{3}(b \otimes c)=0, f_{3}(a \otimes a)=\lambda a, f_{3}(b \otimes b)=\gamma b$ and $f_{3}(c \otimes c)=\mu c$.

(27) There exists $(\lambda, \gamma) \in\left(\mathbb{K}^{*}\right)^{2}$ such that $f_{3}(a \otimes b)=0, f_{3}(a \otimes c)=0, f_{3}(b \otimes c)=$ $0, f_{3}(a \otimes a)=\lambda c, f_{3}(b \otimes b)=\gamma c$ and $f_{3}(c \otimes c)=0$.

(28) There exists $(\lambda, \gamma) \in\left(\mathbb{K}^{*}\right)^{2}$ such that $f_{3}(a \otimes b)=0, f_{3}(a \otimes c)=0, f_{3}(b \otimes c)=$ $0, f_{3}(a \otimes a)=\lambda c, f_{3}(b \otimes b)=\gamma b$ and $f_{3}(c \otimes c)=0$. 
(29) There exists $(\lambda, \gamma) \in\left(\mathbb{K}^{*}\right)^{2}$ such that $f_{3}(a \otimes b)=0, f_{3}(a \otimes c)=0, f_{3}(b \otimes c)=$ $0, f_{3}(a \otimes a)=\lambda a, f_{3}(b \otimes b)=\gamma b$ and $f_{3}(c \otimes c)=0$.

(30) There exists $\lambda \in \mathbb{K}^{*}$ such that $f_{3}(a \otimes b)=0, f_{3}(a \otimes c)=0, f_{3}(b \otimes c)=0$, $f_{3}(a \otimes a)=\lambda b, f_{3}(b \otimes b)=0$ and $f_{3}(c \otimes c)=0$.

(31) There exists $\lambda \in \mathbb{K}^{*}$ such that $f_{3}(a \otimes b)=0, f_{3}(a \otimes c)=0, f_{3}(b \otimes c)=0$, $f_{3}(a \otimes a)=\lambda a, f_{3}(b \otimes b)=0$ and $f_{3}(c \otimes c)=0$.

(32) The map $f_{3}$ is the null map.

Proof. We use the fact that the map $f_{3}$ is associative and commutative, and then, we get the lemma by direct quite long calculations.

Proposition 5.7. Let $X=\{a, b, c\}$ be an alphabet of cardinality 3 and let $\square$ be a weak stuffle product. In the previous lemma, if $f_{3}$ satisfies one of the items (1), (2), (5), (6), (8), (14), (15) then either $\left(f_{1} \equiv 0\right.$ and $\left.f_{2} \equiv 0\right)$ or $\left(f_{1}(a \otimes b)=1\right.$ and $f_{2}(a \otimes b)=1$ for $\left.(a, b) \in X^{2}\right)$.

\section{Weak stuffle product and Hopf algebras}

If $\square$ is the classical shuffle product or the classical stuffle product then the algebra $(\mathbb{K}\langle X\rangle, \square)$ can be equipped with a compatible coalgebra structure, thanks to the deconcatenation coproduct, which makes it into a Hopf algebra. Are there other weak stuffle products compatible with the deconcatenation? We begin by recalling the Hopf algebra construction for stuffle algebras given in $[16,18,17]$. We then turn to the case of weak stuffle algebras.

Theorem 6.1. Let $X$ be a countable alphabet, let $\mathbb{K}\langle X\rangle$ be the vector space generated by words on the alphabet $X$. We assume there exists at least one product $\diamond$ on $\mathbb{K} . X$ which is commutative and associative. We define the product $\star$ and the coproduct of deconcatenation $\Delta$ by:

$$
a u \star b v=a(u \star b v)+b(a u \star v)+(a \diamond b)(u \star v)
$$

and

$$
\Delta(w)=\sum_{\substack{(u, v) \in(\mathbb{K}\langle X\rangle)^{2}, u v=w}} u \otimes v
$$

for any letters $a$ and $b$ and any words $u, v$ and $w$.

Then $(\mathbb{K}\langle X\rangle, \star, \Delta)$ is a Hopf algebra.

Proof. This theorem is proven in $[16,18,17]$ by induction and using the filtration given by the length of words. 
Theorem 6.2. Let $X$ be a countable alphabet of cardinality $n \in \mathbb{N} \cup\{+\infty\}$ and let $\square$ be a weak stuffle product on $\mathbb{K}\langle X\rangle$. We denote by $\Delta$ the deconcatenation coproduct. If $\Delta$ respects $\square$ (i.e. if $\Delta$ is an algebra morphism) then the underlying weak shuffle product is the classical shuffle product.

Proof. Let $\square$ be a weak stuffle product. We assume the deconcatenation respects $\square$. Then, for any distinct letters $a$ and $b$ :

$$
\begin{aligned}
\Delta(a \square a)= & \left(f_{1}(a \otimes a)+f_{2}(a \otimes a)\right) \Delta(a a)+\Delta\left(f_{3}(a \otimes a)\right) \\
= & \left(f_{1}(a \otimes a)+f_{2}(a \otimes a)\right) \Delta(a a)+k(a \otimes a) \Delta(g(a \otimes a)) \\
= & \left(f_{1}(a \otimes a)+f_{2}(a \otimes a)\right)(a a \otimes 1+a \otimes a+1 \otimes a a) \\
& +k(a \otimes a)(g(a \otimes a) \otimes 1+1 \otimes g(a \otimes a)) \\
= & \Delta(a) \square \Delta(a) \\
= & \left(f_{1}(a \otimes a)+f_{2}(a \otimes a)\right)(a a \otimes 1+1 \otimes a a)+2 a \otimes a \\
& +k(a \otimes a)(g(a \otimes a) \otimes 1+1 \otimes g(a \otimes a)), \\
\Delta(a \square b)= & f_{1}(a \otimes b) \Delta(a b)+f_{1}(b \otimes a) \Delta(b a)+k(a \otimes b) \Delta(g(a \otimes b)) \\
= & f_{1}(a \otimes b)(a b \otimes 1+a \otimes b+1 \otimes a b)+f_{1}(b \otimes a)(b a \otimes 1+b \otimes a+1 \otimes b a) \\
& +k(a \otimes b)(g(a \otimes b) \otimes 1+1 \otimes g(a \otimes b)) \\
= & \Delta(a) \square \Delta(b)=f_{1}(a \otimes b)(a b \otimes 1+1 \otimes a b)+f_{1}(b \otimes a)(b a \otimes 1+1 \otimes b a)+a \otimes b+b \otimes a \\
& +k(a \otimes b)(g(a \otimes b) \otimes 1+1 \otimes g(a \otimes b)) .
\end{aligned}
$$

So, $f_{1}(a \otimes a)=f_{2}(a \otimes a)=f_{1}(a \otimes b)=f_{1}(b \otimes a)=1$.

The reversal is a particular case of Theorem 6.1.

\section{Computation programs}

We give computation programs realised to compute the weak shuffle of two words or to prove Lemma 3.17. In the sequel we assume the alphabet $X$ is the set of integers $\{1, \ldots, c\}$ and a word is a list $\left[i_{1}, \ldots, i_{n}\right]$.

We first present a function which computes the weak shuffle product of two words. This function, called weak_shuffle_product, takes as entries a list Rules which coresponds to the values taken by $f_{1}$ and $f_{2}$ and two lists w1 and w 2 which represent the two words to use for computations. We assume

$$
\begin{aligned}
\text { Rules }= & {\left[f_{1}(1 \otimes 2), \ldots, f_{1}(1 \otimes c), \ldots f_{1}(c \otimes 1), \ldots, f_{1}(c \otimes c-1),\right.} \\
& \left.f_{1}(1 \otimes 1), f_{2}(1 \otimes 1), \ldots, f_{1}(c \otimes c), f_{2}(c \otimes c)\right] .
\end{aligned}
$$


As exit, the function return a list. Each element of the result is a list of two elements A and B: A is the number of times the word represented by B appears in the weak shuffle product of $\mathrm{w} 1$ and $\mathrm{w} 2$.

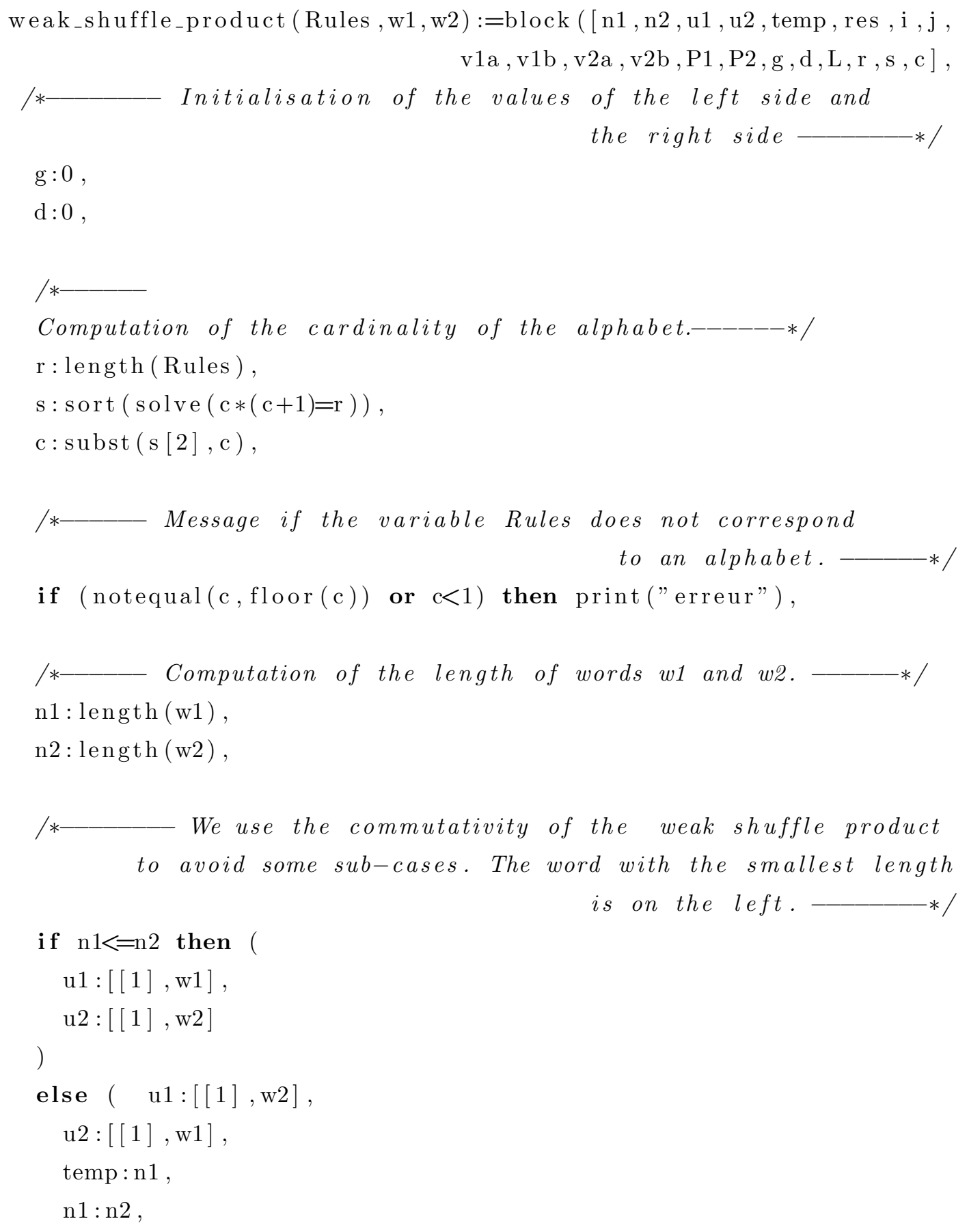




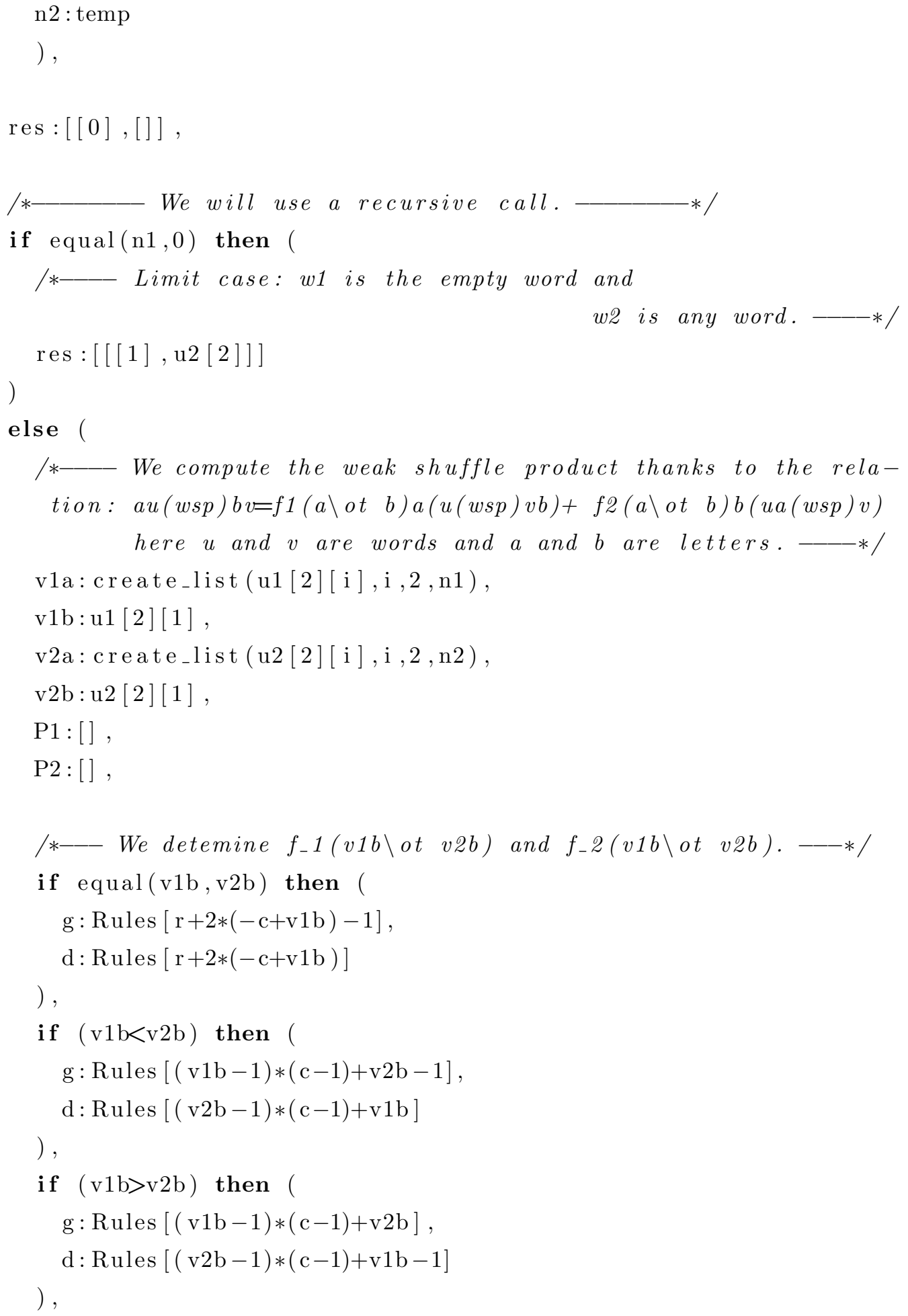




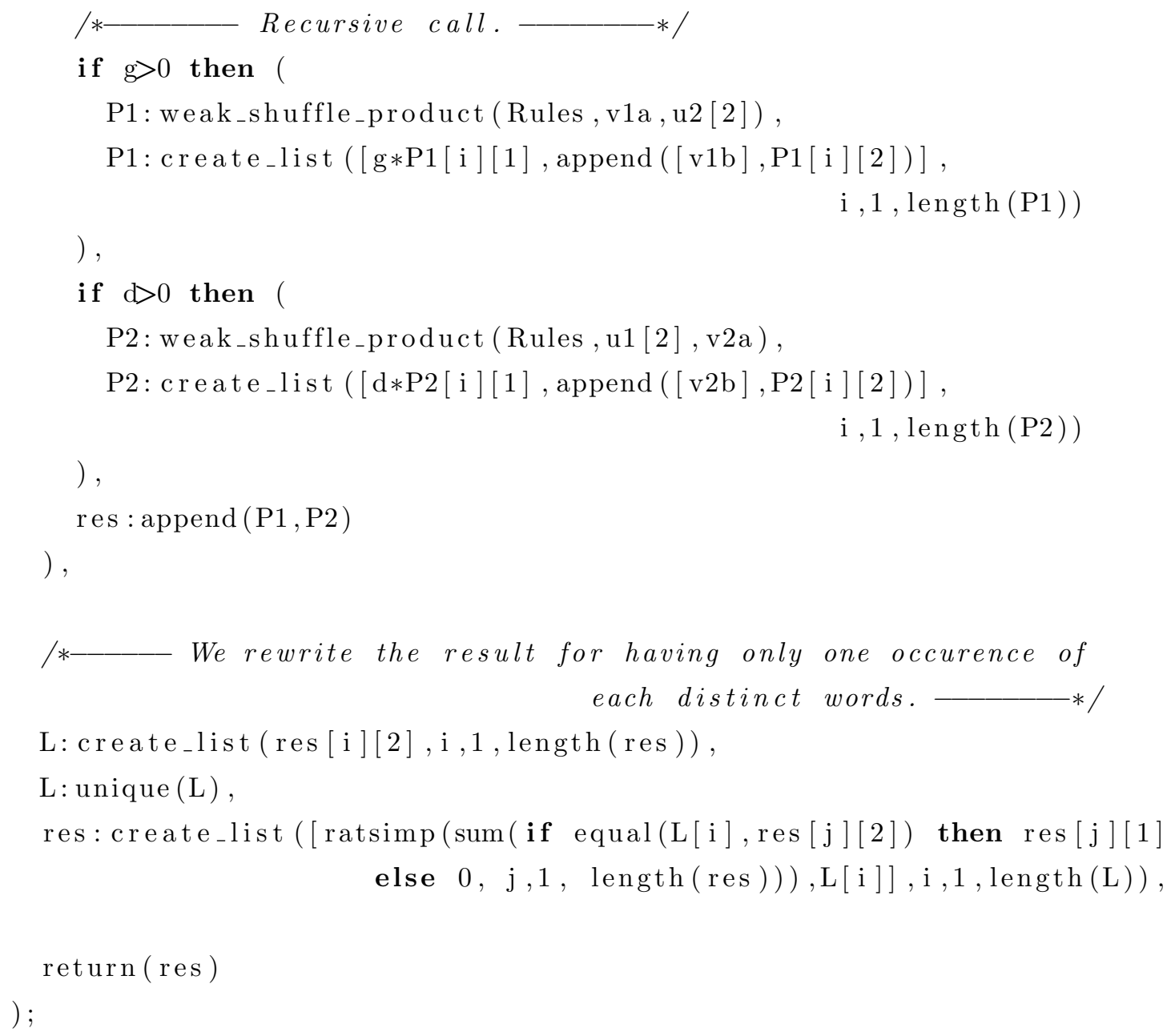

In the sequel, the functions aim at proving if the following statement is true or not for some low $n$. Let $n$ be a positive integer and let $w_{1}, w_{2}$ and $w$ be three non-empty words of length $n$ such that $w_{1} \leq w_{2} \leq w$ and $w_{1}<w$. Then $\max \left(w_{1} \square{ }_{9} w_{2}\right)<\max (w \square \underset{9}{\square} w)$ ? It is trivial for $n=1$. For $n=2$, it comes from computations doing in the proof of Proposition 3.14. Thus, those cases are not treated.

The function words aims at building all words of length $n$ with an alphabet of cardinality $c$. It takes as entries the integers $n$ and $c$ and returns a list where each element is a list coresponding to a word. In the result, words are ordered by the ascending order.

$\operatorname{words}(\mathrm{n}, \mathrm{c}):=\operatorname{block}([\operatorname{res}, \mathrm{i}, \mathrm{j}, \mathrm{U}]$, 


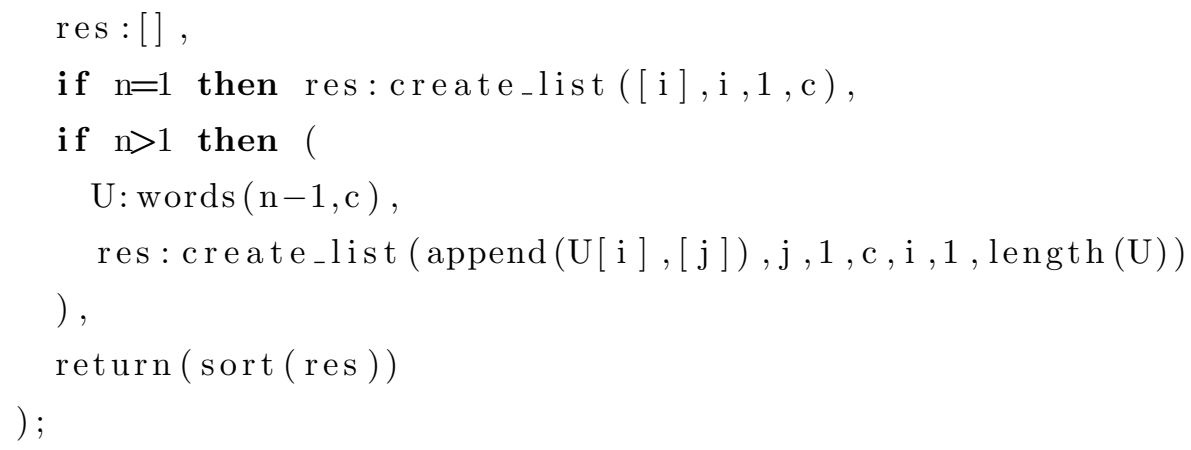

The function spectrum_product aims at determining words appearing in the weak shuffle product of two words w1 and w2. It takes as entries a list Rules which gives the rules of computation for the weak shuffle product, an integer $r$ which is the length of the list Rules, an integer $c$ which is the cardinality of the alphabet, and two lists w1 and w2 which represent the two words to use for computations.

As exit, the function return a list ordered thanks to the ascending order where each element is a list representing a word appearing in the weak shuffle product of two words w1 and w2.

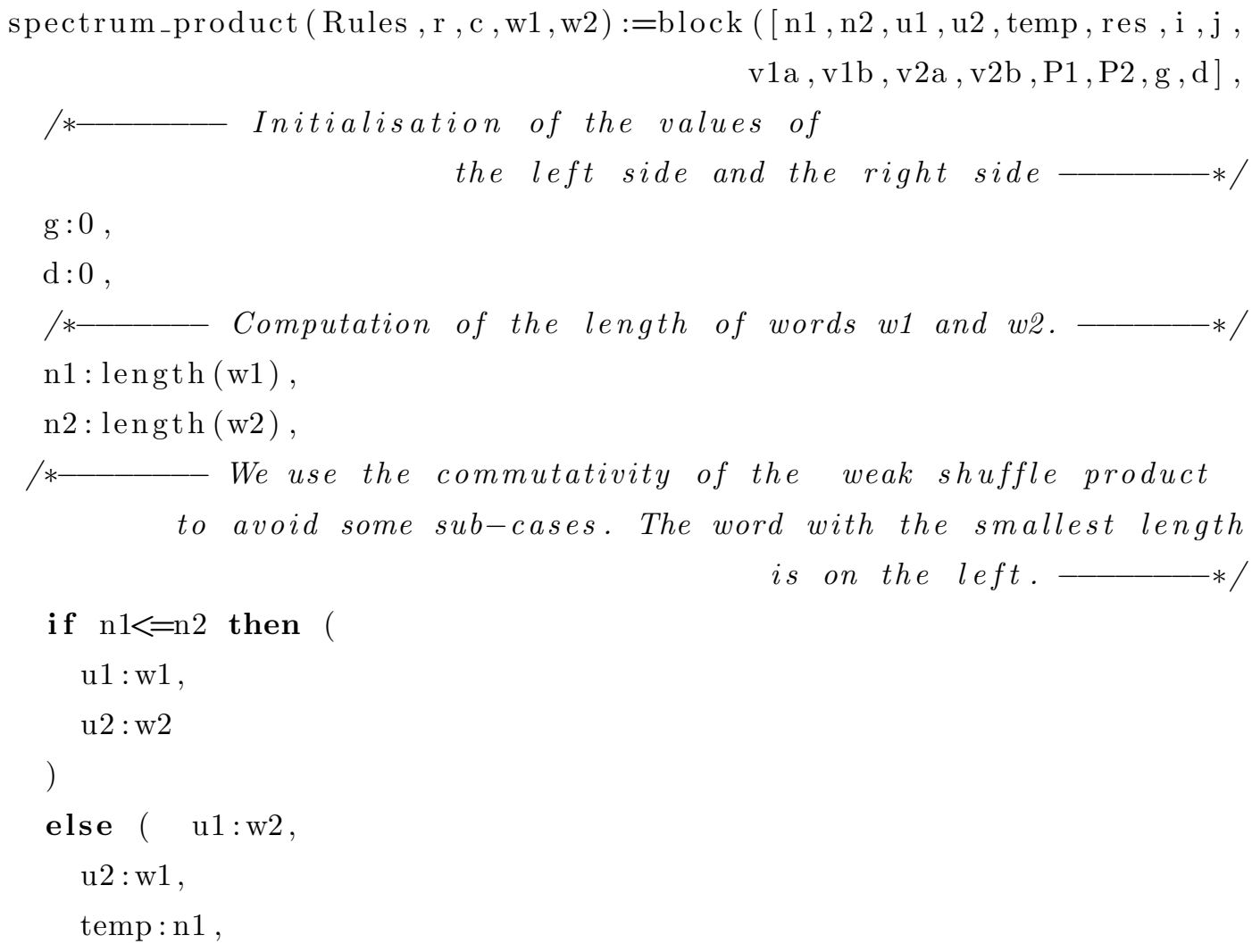




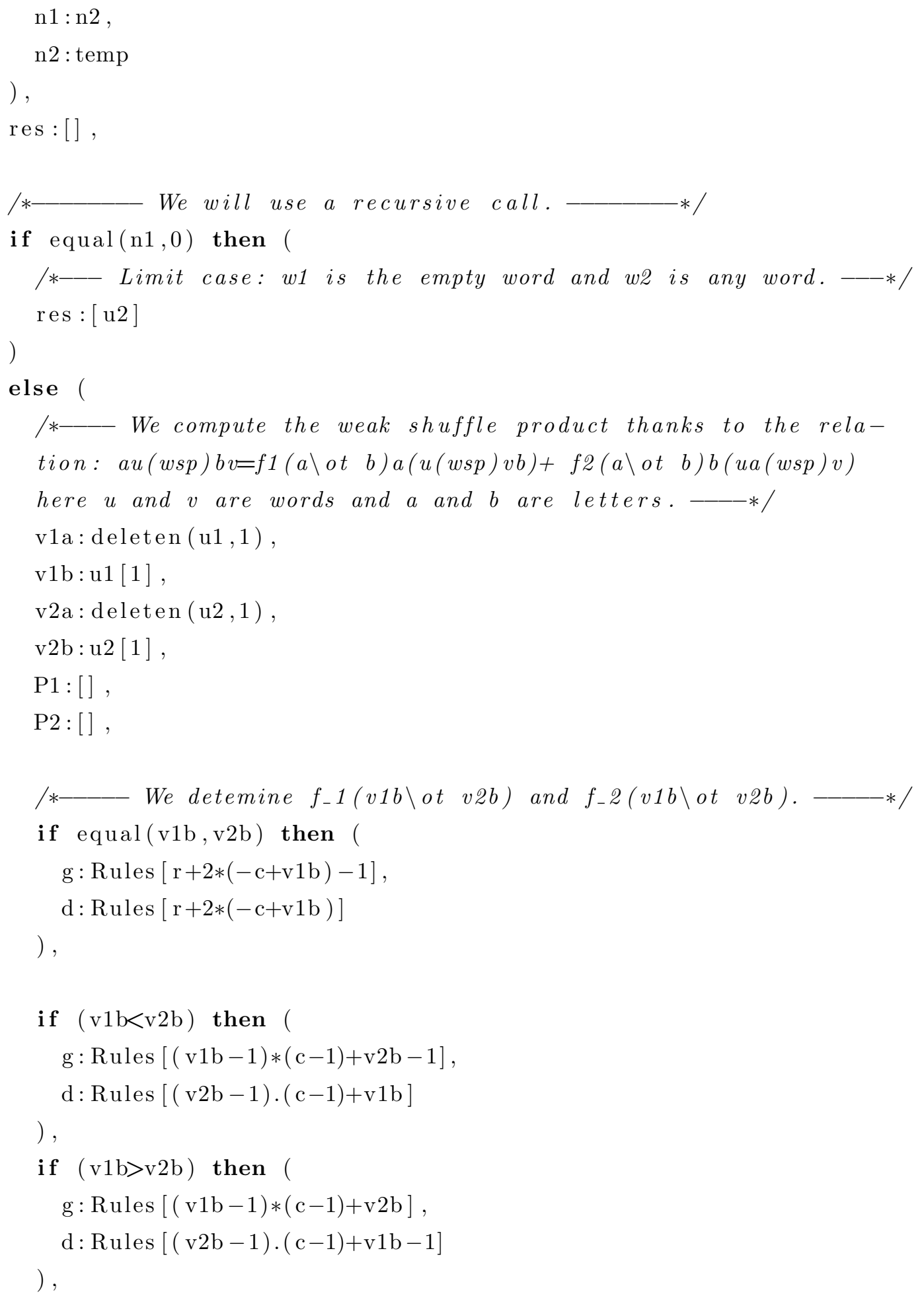




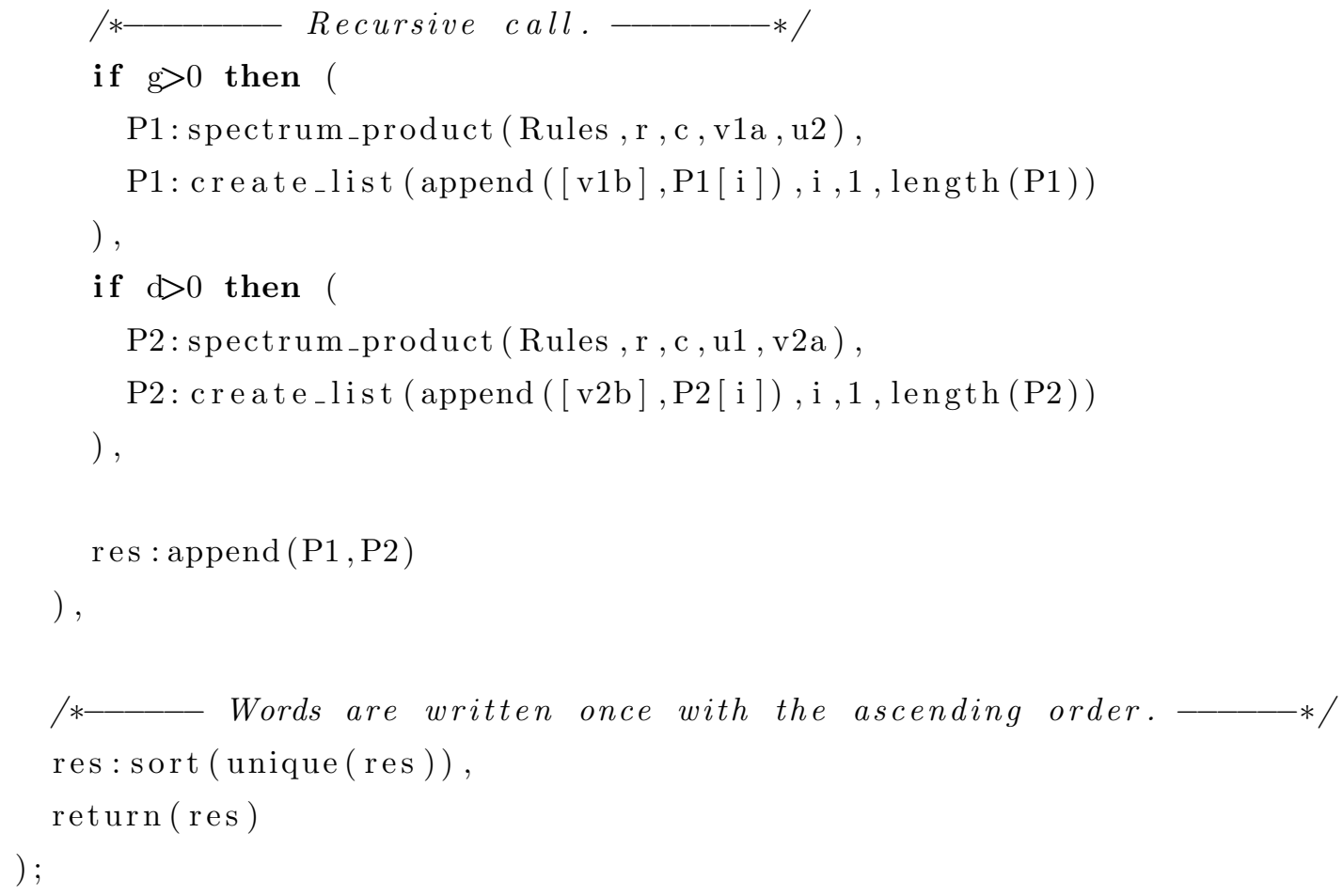

The function maximum_product takes as entries a list Rules corresponding to the weak shuffle product, an integer $r$ which is the length of Rules, an integer $c$ which is the cardinality of the alphabet, an integer $n$ which is the length of words used, a list $W$ which represents the list of words of length $n$, an integer $l$ which is the length of $W$, an integer $k$ which is the level of computation. The function returns a list of length $k-5$. The first one is a list of only one element which is $\max (W[6] \underset{9}{\square} W[6])$. In the result, the element $p$ with $2 \leq p \leq k-5$ is a list of two elements $A_{p}$ and $B_{p}$ where $A_{p}=\max \left(\max \left(w_{1} \underset{9}{\square} w_{2}\right)\right)$ with $w_{1}<W[p]$ and $w_{2} \leq W[k]$ and $B_{p}=\max (W[p] \underset{9}{\square} W[p])$. This function really depends on the weak shuffle product $\underset{9}{\square}$.

maximum_product (Rules, r , c , n,W, l, k) :=block ( [ res , i , P, in it ],

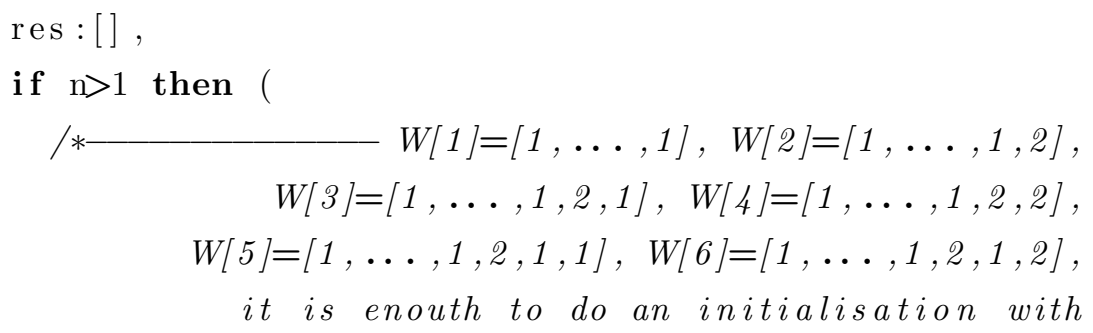




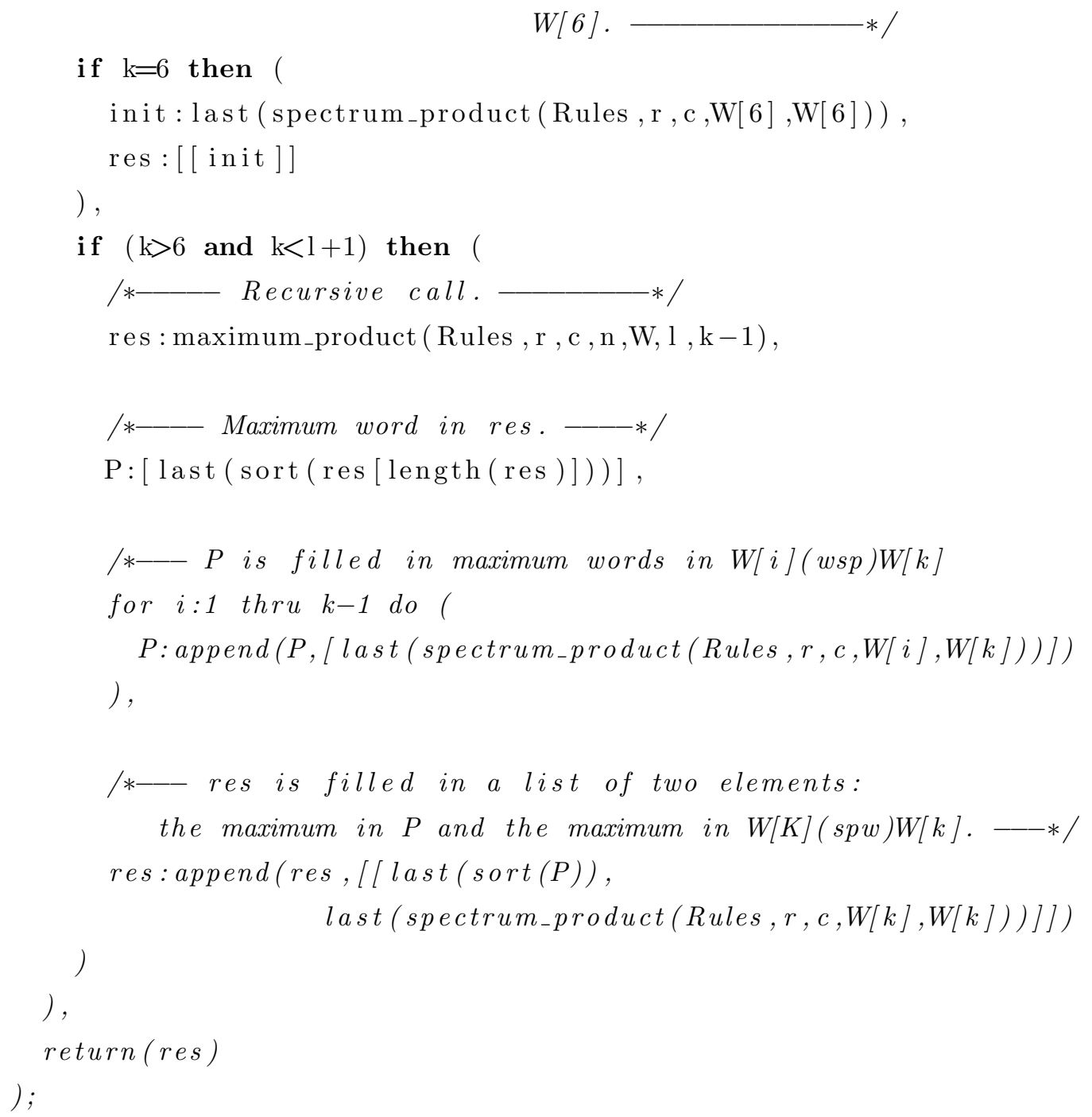

The function proof_statement determines if the statement given at the beginning of the section is proved for words of length $n$. As entries, it takes a list Rules corresponding to the weak shuffle product and an integer coresponding to the length of words used. It returns a boolean. The boolean is true if the statement if satisfied and false if the statement is not satisfied. Since this function uses maximum_product, it depends on the weak shuffle product $\underset{9}{\square}$.

proof_statement (Rules, n):=block ([res, P, U, i , p, c, r, s, W, l] , /*-_ Computation of the cardinality of the alphabet. - - * / r: length (Rules), 


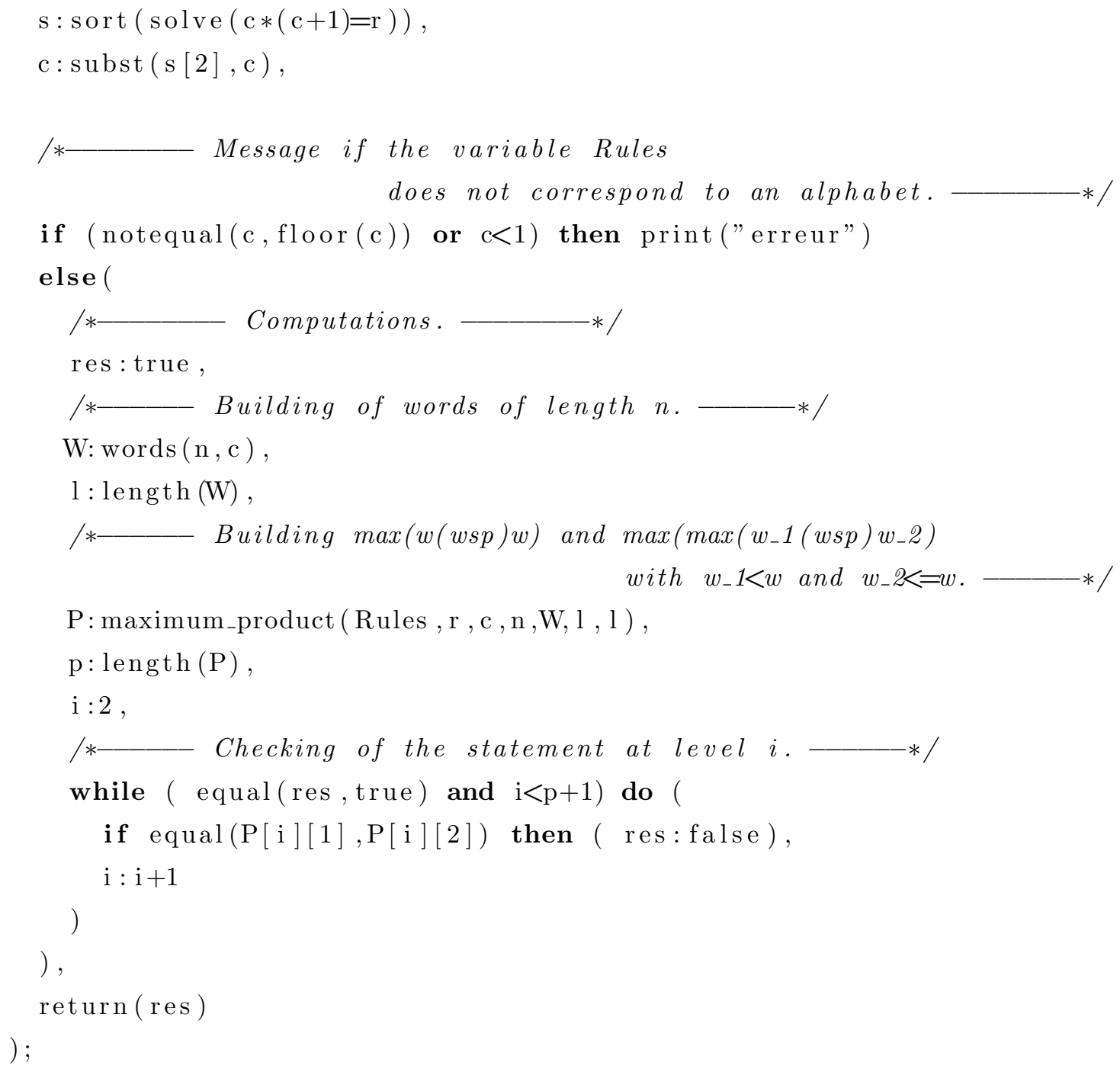

Acknowledgement: I would like to thank the anonymous referees for their useful comments and suggestions. I would like to thank all peaple who supported me for this work. This work was funding by the Laboratoire Paul Painlev at Universit de Lille, the Fdration de Recherche Mathmatique des Hauts-de-France, the ANR Alcohol project ANR-19-CE40-0006 and the Labex CEMPI ANR-11-LABX-000701.

\section{References}

[1] M. Aguiar and J-L. Loday, Quadri-algebras, J. Pure Appl. Algebra, 191(3) (2004), 205-221. 
[2] D. M. Bradley, Multiple q-zeta values, J. Algebra, 283(2) (2005), 752-798.

[3] F. Chapoton, Un thorme de Cartier-Milnor-Moore-Quillen pour les bigbres dendriformes et les algbres braces, J. Pure Appl. Algebra, 168(1) (2002), 1-18.

[4] G. Duchamp, F. Hivert, J.-C. Novelli and J.-Y. Thibon, Noncommutative symmetric functions VII: Free quasi-symmetric functions revisited, Ann. Comb., 15 (2011), 655-673.

[5] G. Duchamp, F. Hivert and J-Y. Thibon, Noncommutative symmetric functions VI: Free quasi-symmetric functions and related algebras, Internat. J. Algebra Comput., 12(5) (2002), 671-717.

[6] K. Ebrahimi-Fard and L. Guo, Mixable shuffles, quasi-shuffles and Hopf algebras, J. Algebraic Combin., 24(1) (2006), 83-101.

[7] K. Ebrahimi-Fard, D. Manchon and J. Singer, Duality and q-multiple zeta values, Adv. Math., 298 (2016), 254-285.

[8] K. Ebrahimi-Fard, D. Manchon and J. Singer, The Hopf algebra of q-multiple polylogarithms with non-positive arguments, Int. Math. Res. Not., 16 (2017), 4882-4922.

[9] L. Foissy, Les algbres de Hopf des arbres enracins dcors. II, Bull. Sci. Math., 126(4) (2002), 249-288.

[10] L. Foissy, Bidendriform bialgebras, trees and free quasi-symmetric functions, J. Pure Appl. Algebra, 209(2) (2007), 439-459.

[11] L. Foissy, Free quadri-algebras and dual quadri-algebras, Comm. Algebra, 48(12) (2020), 5123-5141.

[12] L. Foissy, F. Patras and J.-Y. Thibon, Deformations of shuffles and quasishuffles, Ann. Inst. Fourier, 66(1) (2016), 209-237.

[13] I. M. Gessel, Multipartite p-partitions and inner products of skew Schur functions, Combinatorics and algebra (Boulder, Colo., (1983)), Contemp. Math., 34, Amer. Math. Soc., Providence, RI, (1984), 289-317.

[14] L. Guo and W. Keigher, Baxter Algebras and Shuffle Products, Adv. Math., 150(1) (2000), 117-149.

[15] M. E. Hoffman, The algebra of multiple harmonic series, J. Algebra, 194(2) (1997), 477-495.

[16] M. E. Hoffman, Quasi-shuffle products, J. Algebraic Combin., 11(1) (2000), 49-68.

[17] M. E. Hoffman, Quasi-shuffle algebras and applications, In: Chapoton, F., et al. (eds.) Algebraic Combinatorics, Resurgence, Moulds and Applications, Vol. 2 (IRMA Lectures in Mathematics and Theoretical Physics vol. 32), European Math. Soc. Publ. House, Berlin (2020), 327-348. 
[18] M. E. Hoffman and K. Ihara, Quasi-shuffle products revisited, J. Algebra, 481 (2017), 293-326.

[19] M. E. Hoffman and Y. Ohno, Relations of multiple zeta values and their algebraic expression, J. Algebra, 262(2) (2003), 332-347.

[20] R-Q. Jian, Quantum quasi-shuffle algebras II, J. Algebra, 472 (2017), 480-506.

[21] R-Q. Jian, M. Rosso and J. Zhang, Quantum Quasi-Shuffle Algebras, Lett. Math. Phys., 92(1) (2010), 1-16.

[22] J-L. Loday, Dialgebras, in Dialgebras and related operads, Lecture Notes in Math., Springer, Berlin, 1763 (2001), 7-66.

[23] J-L. Loday and M. Ronco, Hopf algebra of the planar binary trees, Adv. Math., 139(2) (1998), 293-309.

[24] C. Malvenuto, Produits et Coproduits des Fonctions Quasi-Symtriques et de l'algbre des Descentes, Ph.D. thesis, Universit du Qubec Montral, Laboratoire de Combinatoire et d'Informatique Mathmatique, 1994.

[25] C. Malvenuto and C. Reutenauer, Duality between quasi-symmetric functions and the Solomon descent algebra, J. Algebra, 177(3) (1995), 967-982.

[26] C. Mammez, A propos de l'algbre de Hopf des mots tasss WMat, Bull. Sci. Math., 145 (2018), 53-96.

[27] M. Ronco, A Milnor-Moore theorem for dendriform Hopf algebras, C. R. Acad. Sci. Paris Sr. I Math., 332(2) (2001), 109-114.

[28] K.-G. Schlesinger, Some remarks on q-deformed multiple polylogarithms, arXiv:math/0111022, (2001).

[29] J. Singer, On q-analogues of multiple zeta values, Funct. Approx. Comment. Math., 53(1) (2015), 135-165.

[30] J. Singer, On Bradley's q-MZVs and a generalized Euler decomposition formula, J. Algebra, 454 (2016), 92-122.

[31] J. Singer, $q$-Analogues of Multiple Zeta Values and Their Application in Renormalization, Ph.D. thesis, Der Naturwissenschaftlichen Fakultt, der Friedrich-Alexander-Univedrsitt, 2016.

[32] B. Vallette, Manin products, Koszul duality, Loday algebras and Deligne conjecture, J. Reine Angew. Math., 620 (2008), 105-164.

[33] Y. Vargas, Hopf algebra of permutation pattern functions, 26th International Conference on Formal Power Series and Algebraic Combinatorics, Chicago, United States, (2014), 839-850.

[34] D. Zagier, Values of zeta functions and their applications, First European Congress of Mathematics, Vol. II (Paris, 1992), vol. 120 of Progr. Math., Birkhäuser, Basel, (1994), 497-512. 
[35] J. Zhao, Multiple q-zeta functions and multiple q-polylogarithms, Ramanujan J., 14(2) (2007), 189-221.

[36] V. V. Zudilin, Algebraic relations for multiple zeta values, Russian Math. Surveys, 58(1) (2003), 1-29.

\author{
Cécile Mammez \\ Univ. Lille, UMR 8524 - Laboratoire Paul Painlev, F-59000 Lille, France \\ CNRS, UMR 8524, F-59000 Lille, France \\ e-mail: cecile.mammez@laposte.net
}

